Journal of Computational Physics, Volume 425, January 2021, Article number 109749

DOI:10.1016/j.jcp.2020.109749

\title{
A reduced-dissipation WENO scheme with automatic dissipation adjustment
}

\author{
Javier Fernández-Fidalgo ${ }^{\mathrm{a}, *}$, Luis Ramírez ${ }^{\mathrm{a}}$, Panagiotis Tsoutsanis ${ }^{\mathrm{b}}$, \\ Ignasi Colominas ${ }^{\mathrm{a}}$, Xesús Nogueira ${ }^{\mathrm{a}, *}$ \\ ${ }^{a}$ Group of Numerical Methods in Engineering, Universidade da Coruña, \\ Campus de Elviña, 15071, A Coruña, Spain \\ ${ }^{b}$ Centre for Computational Engineering Sciences, Cranfield University, \\ Cranfield MK43 OAL, United Kingdom
}

\begin{abstract}
In this paper, we propose a novel modification to the WENO-family schemes to reduce its intrinsic dissipation. In this work, we focus on the WENO5 scheme, which is rewritten in terms of a central plus a dissipative part, and then, the dissipation is controlled based on the flow physics. This is achieved by using the automatic dissipation adjustment (ADA) method in an a posteriori approach. This methodology allows us to greatly increase the accuracy of the original scheme at the same time ensure the robustness of REV1: the numerical method. The accuracy and robustness of the proposed scheme are tested by several selected numerical REV2: benchmarks.
\end{abstract}

Keywords: high-order schemes, WENO, compressible flows, adaptive dissipation

\section{Introduction}

Traditionally, in Computational Fluid Dynamics, second-order numerical methods are often preferred in practical calculations, the main reasons being simplicity of implementation and robustness. In the context of compressible flows, the family of Essentially Non Oscillatory reconstruction schemes, circumvents Godunov's theorem by using nonlinear procedures to obtain highorder reconstructions.

*Corresponding author, e-mail: \{javier.fernandez1,xnogueira\}@udc.es 
The key idea of the Essentially Non Oscillatory (ENO) reconstruction [1] is to systematically avoid the use of the discontinuous cells in the reconstruction stencil whenever possible. This is achieved by computing the divided differences [2] of the involved candidate stencils and picking the smoothest one, which corresponds to that with the smallest value of divided differences.

A considerable improvement to the ENO methodology is the Weighted ENO (WENO) approximation $[3,4,5]$. This approach uses a convex combination of all the different stencils that are used in the ENO approximation. This is done in a nonlinear fashion through a set of nonlinear weights called $\omega_{k}$. In [3] the divided differences that are used in the ENO approach are replaced by a more efficient way to compute the relative smoothness of the different stencils via the smoothness detectors $\beta_{k}$, which are a scaled $L_{2}$-norm of the involved polynomials for each stencil.

The original WENO scheme, usually referred to as WENO-JS, EDTR: has several deficiencies that have been pointed out and corrected later on. The first one is that they do not preserve monotonicity, this gave rise to the MPWENO schemes of Balsara et al. [6] that have high phase accuracy and high order of accuracy. The higher-order members of this family are almost spectrally accurate for smooth problems while maintaining robust shock capturing abilities. The second one is that it usually fails to obtain the expected order of accuracy around smooth extrema, where low-order derivatives vanish. In [7] it is pointed out that this fact is due to the excessively slow convergence of the nonlinear weights towards their optimal values. To solve this problem, the authors propose a mapping function that makes the nonlinear weights $\omega_{k}$ to converge faster to their optimal counterparts, reducing the dissipation of the original WENO-JS formulation. This approach, usually referred to as WENO-M is more computationally demanding than the original one, remarkably increasing the computational cost of the numerical simulation.

In [8] it is argued that the improved results of the WENO-M for shockcapturing problems are due to the non-linear adaptation, that is, the nonsmooth stencils should obtain larger weights in order to obtain less-dissipative results. In $[8,9]$ a novel fifth-order WENO scheme, WENO-Z, was proposed by adding a higher-order smoothness indicator $\tau_{5}=\left|\beta_{0}-\beta_{2}\right|$ which is an inexpensive linear combination of the already existing smoothness indicators. This approach is more cost-efficient when compared with the WENO-M and yields similar less-dissipative results. A similar technique, called WENO-Z+, was recently proposed in [10]. This approach increases the relevance of the non-smooth stencils by adding a new term to the WENO-Z weights that uses 
information which is already available in the formulation.

Several other approaches to reduce the excessive dissipation of the WENOJS are worth mentioning. In [11] the strategy is to freeze the weights when the ratio of the largest to the smallest smoothness indicator does not exceed a certain threshold value. Since the smoothness detectors $\beta_{k}$ are not bounded and vary significantly from one test case to another, this threshold is likely to be problem-dependent in order to produce quality results. Another source of dissipation is that of the underlying linear scheme. In [12], a low-dissipation third-order scheme is proposed, this approach considers the addition of a downwind stencil. Another possible approach is to optimize the dispersion-dissipation relation as in [13], or the more general approach of [14] where the authors give a general condition for the relation of dispersion and dissipation on finite difference schemes.

In order to reduce nonlinear adaption while retaining high-order, in [15] a sixth-order hybrid central-upwind WENO-CU6 was introduced and further enhanced with a scale separation method to be of application to turbulence problems [16]. Another recent development that is particularly suitable for DNS and LES of shock-turbulence interactions is a family of Targeted ENO schemes of $[17,18,19]$ coupled with an improved scale separation procedure based on the one described in [16]. The main difference of these schemes with respect to the original WENO formulation is that the nonlinear weights are either applied with the corresponding optimal value or entirely eliminated (ENO-like stencil selection). This approach preserves the robustness and shock-capturing capabilities of the scheme while limiting the numerical dissipation to that of the underlying linear scheme. The optimization proposed in [14] is used to limit the numerical dissipation, losing one order of accuracy of the scheme. Other hybrid approaches such as [20, 21, 22] propose to combine a WENO scheme with a fixed-stencil finite difference scheme, applying the former only in regions with shocks. EDTR: Yet another form of hybridization is the one proposed by the adaptive order WENO schemes of [23], where a higher order centered stencil is combined with lower order stencils in a nonlinear fashion. They have also optimized the classical finite difference WENO schemes by using Legendre polynomial basis and writing the smoothness indicators as perfect squares, which reduces the computational requirements as the order of the WENO scheme scales up.

Another approach within the WENO family is to use a compact reconstruction scheme as in [24]. This approach uses the same smoothness indicators and weighting system as the WENO-M approach, but uses compact 
candidate stencils that relate neighboring interfaces. By doing so, a blocktridiagonal system of equations needs to be solved in order to obtain the reconstructed values. Because of using implicit stencils, in [24] is argued that the compact WENO5 (CRWENO5) achieves higher resolution by using an smaller stencil when compared to the explicit WENO5, however the compact scheme needs boundary closures for non-periodic boundary conditions (usually in the form of the explicit WENO5), and since it uses the same smoothness indicators as the WENO5 for the stencils, in practice the stencil width remains the same as in the explicit WENO scheme.

In this paper, we propose a novel modification to the WENO scheme to reduce its intrinsic dissipation. The WENO5 scheme is rewritten in terms of a central plus a dissipative part, and then, the dissipation is controlled based on the energy ratio $[25,26]$. The idea is that the dissipation of the WENO scheme is adapted to the physics of the flow. The a posteriori approach of $[27,28]$ is used in order to ensure the robustness of the approach.

The structure of this paper is as follows: firstly the governing equations are presented. Next we review the WENO reconstruction and perform a dissipation analysis of the WENO5 scheme, splitting the dissipation from the central part. Afterwards we review the ADA method and propose an extension for compressible flows of the original incompressible formulation. Finally, the mathematical formulation for the WENO5 ADA scheme is presented along with some numerical examples. Finally, conclusions are drawn.

\section{Governing equations}

For this paper, the compressible Navier-Stokes equations in non-dimensional conservative variables will be considered, as in [29]. This system can be expressed in vector form as:

$$
\frac{\partial \mathbf{U}}{\partial t}+\frac{\partial \mathbf{F}}{\partial x}+\frac{\partial \mathbf{G}}{\partial y}+\frac{\partial \mathbf{H}}{\partial z}=\frac{\partial \mathbf{F}_{\mathbf{v}}}{\partial x}+\frac{\partial \mathbf{G}_{\mathbf{v}}}{\partial y}+\frac{\partial \mathbf{H}_{\mathbf{v}}}{\partial z} .
$$

The vector quantities are defined as:

$$
\mathbf{U}=\left(\begin{array}{c}
\rho \\
\rho u \\
\rho v \\
\rho w \\
\rho E
\end{array}\right) \quad \mathbf{F}=\left(\begin{array}{c}
\rho u \\
\rho u^{2}+p \\
\rho u v \\
\rho u w \\
(\rho E+p) u
\end{array}\right) \quad \mathbf{G}=\left(\begin{array}{c}
\rho v \\
\rho v u \\
\rho v^{2}+p \\
\rho v w \\
(\rho E+p) v
\end{array}\right) \quad \mathbf{H}=\left(\begin{array}{c}
\rho w \\
\rho w u \\
\rho w v \\
\rho w^{2}+p \\
(\rho E+p) w
\end{array}\right)
$$




$$
\mathbf{F}_{\mathbf{v}}=\left(\begin{array}{c}
0 \\
\tau_{x x} \\
\tau_{x y} \\
\tau_{x z} \\
\tau_{x k} u_{k}-q_{x}
\end{array}\right) \quad \mathbf{G}_{\mathbf{v}}=\left(\begin{array}{c}
0 \\
\tau_{y x} \\
\tau_{y y} \\
\tau_{y z} \\
\tau_{y k} u_{k}-q_{y}
\end{array}\right) \quad \mathbf{H}_{\mathbf{v}}=\left(\begin{array}{c}
0 \\
\tau_{z x} \\
\tau_{z y} \\
\tau_{z z} \\
\tau_{z k} u_{k}-q_{z}
\end{array}\right)
$$

where the repeated index $k$ on equation (3) stands for Einstein's summation over $x, y$ and $z$. Vector $\mathbf{U}$ is called the conserved variables vector, whereas $\mathbf{F}$, $\mathbf{G}$ and $\mathbf{H}$ are called the $x-, y-$ and $z$-inviscid fluxes respectively. On the RHS are the viscous fluxes denoted as $\mathbf{F}_{\mathbf{v}}, \mathbf{G}_{\mathbf{v}}$ and $\mathbf{H}_{\mathbf{v}}$, that depend on the conserved variables vector and its gradient. The physical quantities involved are the mass density $\rho$, the Cartesian components of the velocity vector $\mathbf{V}=(u, v, w)^{T}$ (also expressed as $\left(u_{x}, u_{y}, u_{z}\right)^{T}$ for abbreviated summation purposes), the pressure $p$ and $\rho E=\frac{p}{\gamma-1}+\frac{1}{2} \rho\|\mathbf{V}\|^{2}$ the total energy per unit volume. The non-dimensional coefficient $\gamma$ is the ratio of specific heat coefficients of the gas/fluid (for an ideal, monatomic gas, $\gamma=7 / 5$ ). These conserved quantities arise naturally from the application of the fundamental laws of conservation of mass, REV2: Newton's Second Law and the law of conservation of energy.

The flow variables have been non-dimensionalized, denoted with a star $\left(^{*}\right)$ superscript, by their corresponding free-stream values, denoted with an $\infty$ subscript, as

$$
\rho^{*}=\frac{\rho}{\rho_{\infty}}, p^{*}=\frac{p}{\rho_{\infty} V_{\infty}^{2}}, \mathbf{V}^{*}=\frac{\mathbf{V}}{V_{\infty}}, \mathbf{x}^{*}=\frac{\mathbf{x}}{L}, t^{*}=\frac{t}{L / V_{\infty}}, T^{*}=\frac{T}{T_{\infty}}, \mu^{*}=\frac{\mu}{\mu_{\infty}} .
$$

The important flow parameters are the Reynolds number $\operatorname{Re}_{\infty}=\frac{\rho_{\infty} V_{\infty} L}{\mu_{\infty}}$ and REV2: the free-stream Mach number $M_{\infty}=V_{\infty} \sqrt{\frac{\rho_{\infty}}{\gamma p_{\infty}}}$. The reference length $L$ will be equal to 1 , unless otherwise noted.

The viscous stress tensor and the heat flux vector are non-dimensionalized as

$$
\begin{gathered}
\boldsymbol{\tau}^{*}=\frac{\mu^{*}}{\operatorname{Re}_{\infty}}\left[-\frac{2}{3}\left(\operatorname{div}^{*} \mathbf{V}^{*}\right) \mathbf{I}+\operatorname{grad}^{*} \mathbf{V}^{*}+\left(\operatorname{grad}^{*} \mathbf{V}^{*}\right)^{T}\right] \\
\mathbf{q}^{*}=-\frac{\mu^{*}}{(\gamma-1) \operatorname{Re}_{\infty} M_{\infty}^{2} \operatorname{Pr}} \operatorname{grad}^{*} T^{*}
\end{gathered}
$$


For convenience, the star $\left(^{*}\right)$ notation will be dropped. The Prandtl number $\operatorname{Pr}=0.72$ is supposed constant. The ideal non-dimensional equation of state is used:

$$
p=\rho R T=\frac{\rho T}{\gamma M_{\infty}^{2}} .
$$

\section{Review on the WENO reconstruction}

REV1: In this work, we will focus on the fifth-order WENO scheme with the mappings proposed in [Y], since it achieves less dissipative results than the original from Jiang and Shu [3] as it was explained in the introduction. But it is important to explain the WENO framework in detail, since the new methodology presented here takes advantage of some of its properties. The philosophy behind the WENO family is to obtain a high-order reconstruction

of the flux at the interfaces $x_{i \pm 1 / 2}$, that is $f_{i \pm 1 / 2}$, and compute the derivative of the flux at each grid point $x_{i}=i \Delta x$ as

$$
\frac{\partial f}{\partial x}\left(x_{i}\right) \approx \frac{f_{i+1 / 2}-f_{i-1 / 2}}{\Delta x} .
$$

REV1: The conservative approximation to the derivative in equation (8) is of high-order provided the flux reconstruction at the interfaces $f_{i \pm 1 / 2}$ is obtained using a high-order scheme, as explained in [3, 30]. To this end, the general form of a finite difference $(2 r-1)$ th-order WENO scheme reads

$$
f_{i+1 / 2}=\sum_{k=1}^{r} \omega_{k} f_{i+1 / 2}^{k},
$$

where the candidate stencils $f_{i+1 / 2}^{k}$ depend on a set of neighboring points $\left(f_{i+k-r}, \ldots, f_{i+k-1}\right)$ and $r$ stands for the number of candidate stencils of $r$-th order that the scheme uses. Each of the fluxes obtained by interpolation in the $k$-th substencil are represented as $f_{i+1 / 2}^{k}$, and $\omega_{k}$ are the corresponding nonlinear weights associated with each substencil. REV1: For the particular case of the WENO5 $(r=3)$, the candidate stencils, portrayed in figure 1 , are

$$
\begin{aligned}
& f_{i+1 / 2}^{1}=\frac{1}{3} f_{i-2}-\frac{7}{6} f_{i-1}+\frac{11}{6} f_{i} \\
& f_{i+1 / 2}^{2}=\frac{-1}{6} f_{i-1}+\frac{5}{6} f_{i}+\frac{1}{3} f_{i+1} . \\
& f_{i+1 / 2}^{3}=\frac{1}{3} f_{i}+\frac{5}{6} f_{i+1}-\frac{1}{6} f_{i+2}
\end{aligned}
$$




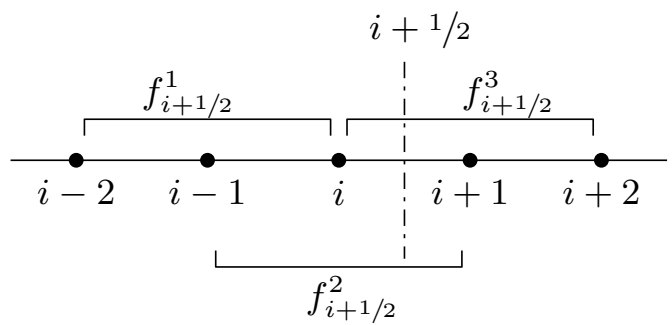

Figure 1: Sketch of the original and present REV1: WENO5 approaches.

There exist optimal weights $\left\{c_{k}\right\}$, with $k=1, \ldots, r$, such that if $\omega_{k}=$ $c_{k} \forall k$, a $(2 r-1)$-th accurate reconstruction is obtained. If this situation happens, the retrieved scheme is called the underlying scheme, and corresponds to a $(2 r-1)$-th upwind biased finite difference scheme. Otherwise, the scheme behaves in a non-oscillatory way by adapting the nonlinear weights so that the smoothest stencils obtain larger weights through the smoothness indicators. REV1: The WENO5 optimal values are $c_{1}=0.1, c_{2}=0.6, c_{3}=0.3$.

The smoothness indicators, $\beta_{k}$ as stated in [31], correspond to a square sum of scaled $L_{2}$ norms of all the derivatives of the interpolating polynomial over the cell taken into consideration, as it can be seen in equation (11).

$$
\beta_{k}=\sum_{l=1}^{r-1} \Delta x^{2 l-1} \int_{x_{i-\frac{1}{2}}}^{x_{i+\frac{1}{2}}}\left(\frac{d^{l}}{d x^{l}} p_{k}(x)\right)^{2} d x .
$$

REV1: In the particular case of the WENO5 $(r=3)$, the smoothness indicators can be expressed as

$$
\begin{aligned}
& \beta_{1}=\frac{13}{12}\left(f_{i-2}-2 f_{i-1}+f_{i}\right)^{2}+\frac{1}{4}\left(f_{i-2}-4 f_{i-1}+3 f_{i}\right)^{2} \\
& \beta_{2}=\frac{13}{12}\left(f_{i-1}-2 f_{i}+f_{i+1}\right)^{2}+\frac{1}{4}\left(f_{i-1}-f_{i+1}\right)^{2} \\
& \beta_{3}=\frac{13}{12}\left(f_{i}-2 f_{i+1}+f_{i+2}\right)^{2}+\frac{1}{4}\left(3 f_{i}-4 f_{i+1}+f_{i+2}\right)^{2} .
\end{aligned}
$$

The optimal weights REV1: $c_{k}$ are then scaled according to $\beta_{k}$ as

$$
\alpha_{k}=\frac{c_{k}}{\left(\beta_{k}+\varepsilon\right)^{m}}
$$


where, in the original WENO-JS [3], $m=2$ and $\varepsilon$ is a small number to prevent division by zero, usually 1E-6. To guarantee the convexity of the weights $\left(\omega_{k}>0 \quad \forall k\right)$, the weights $\alpha_{k}$ are normalized as:

$$
\omega_{k}=\frac{\alpha_{k}}{\sum_{l=1}^{r} \alpha_{l}} .
$$

REV1: Once the candidate stencils and the nonlinear weights have been defined, using equation (9) the value at the interface can be computed with the desired accuracy.

REV1: As commented in the introduction, instead of using the original WENO-JS [3], we will focus on the fifth-order WENO scheme with the mappings proposed in [Y], since it achieves less dissipative results than the original scheme. The only modification is to apply the mapping function $\mathcal{H}_{k}(\omega)$ of equation (15) to each nonlinear weight and renormalize them as in equation (16).

$$
\begin{gathered}
\mathcal{H}_{k}(\omega)=\frac{\omega\left(c_{k}+c_{k}^{2}-3 c_{k} \omega+\omega^{2}\right)}{c_{k}^{2}+\omega\left(1-2 c_{k}\right)} \\
\alpha_{k}^{M}=\mathcal{H}_{k}\left(\omega_{k}\right) \quad \omega_{k}^{M}=\frac{\alpha_{k}^{M}}{\sum_{l=1}^{r} \alpha_{l}^{M}}
\end{gathered}
$$

Computing the mapped nonlinear weights this way, ensures less dissipative results, since the nonlinear adaption is reduced, as explained. REV1: Once the mapped nonlinear weights are defined in equation (16), the resulting flux at the interface, can be computed by substituting the original weights by the mapped ones in equation (9).

\section{Dissipation analysis of the WENO5 scheme}

Any upwind-biased Finite Difference scheme can be separated into a central plus a dissipation part. As an example, let us consider the underlying

scheme for the WENO5, written at an interface for a generic scalar variable $u$ as:

$$
u_{i+1 / 2}=\frac{1}{30} u_{i-2}-\frac{13}{60} u_{i-1}+\frac{47}{60} u_{i}+\frac{9}{20} u_{i+1}-\frac{1}{20} u_{i+2}
$$


The scheme of equation (17) can be written as an even-order, central scheme plus some other terms that conform the dissipative part. This can be re-ordered as:

$$
u_{i+1 / 2}=\underbrace{\frac{1}{12}\left(-u_{i-1}+7 u_{i}+7 u_{i+1}-u_{i+2}\right)}_{\text {central scheme }}+\underbrace{\frac{1}{30}\left(u_{i-2}-4 u_{i-1}+6 u_{i}-4 u_{i+1}+u_{i+2}\right)}_{\text {dissipation }} .
$$

As usual, to compute the derivative, equation (18) can be evaluated at two consecutive interfaces, and by approximating the derivative as in equation (8), yields:

$$
\frac{\partial u}{\partial x} \approx \underbrace{\frac{u_{i-2}-8 u_{i-1}+8 u_{i+1}-u_{i+2}}{12 \Delta x}}_{\text {central scheme }}+\underbrace{\frac{-u_{i-3}+5 u_{i-2}-10 u_{i-1}+10 u_{i}-5 u_{i+1}+u_{i+2}}{30 \Delta x}}_{\text {dissipation }} .
$$

Given the linear properties of the underlying scheme for the WENO5, it is fairly easy to segregate the central and dissipative parts. But a similar analysis can be performed for the non-linear part of the WENO5 scheme for the scalar and vector cases as in [32].

\subsection{Scalar conservation laws}

Let us assume that we need to solve the scalar transport equation

$$
\frac{\partial \phi}{\partial t}+\frac{\partial f}{\partial x}=0
$$

where the flux $f=a \phi$. After splitting the flux $f$, the positive and negative part of the flux approximated at the interface can be written, with the shorthand notation $\Delta f_{k+1 / 2}^{ \pm}=f_{k+1}^{ \pm}-f_{k}^{ \pm}$, as:

$$
\begin{aligned}
& f_{i+1 / 2}^{+}=\frac{1}{12}\left(-f_{i-1}^{+}+7 f_{i}^{+}+7 f_{i+1}^{+}-f_{i+2}^{+}\right)-\varphi_{N}\left(\Delta f_{i-3 / 2}^{+}, \Delta f_{i-1 / 2}^{+}, \Delta f_{i+1 / 2}^{+}, \Delta f_{i+3 / 2}^{+}\right) \\
& f_{i+1 / 2}^{-}=\frac{1}{12}\left(-f_{i-1}^{-}+7 f_{i}^{-}+7 f_{i+1}^{-}-f_{i+2}^{-}\right)+\varphi_{N}\left(\Delta f_{i+5 / 2}^{-}, \Delta f_{i+3 / 2}^{-}, \Delta f_{i+1 / 2}^{-}, \Delta f_{i-1 / 2}^{-}\right)
\end{aligned}
$$

REV2: Taking into account that any WENO scheme satisfies $\sum \omega_{k}=1$, in the WENO5 scheme we can solve for $\omega_{2}=1-\omega_{1}-\omega_{3}$ and define the dissipation function

$$
\varphi_{N}(a, b, c, d)=\frac{1}{3} \omega_{1}(a-2 b+c)+\frac{1}{6}\left(\omega_{3}-\frac{1}{2}\right)(b-2 c+d) .
$$


REV2: Equation (23) obtains the dissipative part of the WENO5 scheme, and is obtained by plugging equation (10) into equation (9) and the subtracting the central part of the flux.

In the end, the reconstructed flux at the interface can be expressed as:

$$
\begin{aligned}
f_{i+1 / 2} & =\frac{1}{12}\left(-f_{i-1}+7 f_{i}+7 f_{i+1}-f_{i+2}\right) \\
& -\varphi_{N}\left(\Delta f_{i-3 / 2}^{+}, \Delta f_{i-1 / 2}^{+}, \Delta f_{i+1 / 2}^{+}, \Delta f_{i+3 / 2}^{+}\right) \\
& +\varphi_{N}\left(\Delta f_{i+5 / 2}^{-}, \Delta f_{i+3 / 2}^{-}, \Delta f_{i+1 / 2}^{-}, \Delta f_{i-1 / 2}^{-}\right)
\end{aligned}
$$

where the same structure of central scheme plus dissipation part can be observed.

\subsection{Systems of conservation laws}

Since the WENO5 scheme is used for the inviscid part of the NavierStokes equations, the same procedure as for the Euler equations can be used. We perform a global Lax-Friedrichs flux splitting procedure on the inviscid fluxes as:

$$
\mathbf{F}^{ \pm}=\frac{1}{2}(\mathbf{F} \pm \alpha \mathbf{U})
$$

where $\alpha=\max _{\mathcal{R}}\left(\max _{m}\left|\lambda^{(m)}\right|\right)$ can be seen as a dissipation coefficient, being $\mathcal{R}$ the stencil that is taken into account around the point in consideration. The $\lambda^{(m)}$ are the eigenvalues of the system, and $\mathcal{R}$ is taken as the whole line (row or column) where the point lies.

Since the WENO5 characteristic-wise approach is employed, the matrices of REV2: eigenvectors are used to transform the vector of conserved variables onto the characteristic space. The left eigenvector matrix $\mathbf{L}_{i+1 / 2}$ and the right eigenvector matrix $\mathbf{R}_{i+1 / 2}$ are inverse of each other. The transformation between the characteristic space and the original space for the fluxes is:

$$
\begin{aligned}
\mathcal{F}_{k} & =\mathbf{L}_{i+1 / 2} \mathbf{F}_{k} \quad k=\{i-2, \ldots, i+2\} \\
\mathbf{F}_{k} & =\mathbf{R}_{i+1 / 2} \mathcal{F}_{k}
\end{aligned}
$$

we end up with the corresponding vector expression: 


$$
\begin{aligned}
\mathbf{F}_{i+1 / 2} & =\frac{1}{12}\left(-\mathbf{F}_{i-1}+7 \mathbf{F}_{i}+7 \mathbf{F}_{i+1}-\mathbf{F}_{i+2}\right) \\
& -\mathbf{R}_{i+1 / 2} \boldsymbol{\varphi}_{N}\left(\Delta \mathcal{F}_{i-3 / 2}^{+}, \Delta \mathcal{F}_{i-1 / 2}^{+}, \Delta \mathcal{F}_{i+1 / 2}^{+}, \Delta \mathcal{F}_{i+3 / 2}^{+}\right) \\
& +\mathbf{R}_{i+1 / 2} \boldsymbol{\varphi}_{N}\left(\Delta \mathcal{F}_{i+5 / 2}^{-}, \Delta \mathcal{F}_{i+3 / 2}^{-}, \Delta \mathcal{F}_{i+1 / 2}^{-}, \Delta \mathcal{F}_{i-1 / 2}^{-}\right)
\end{aligned}
$$

where $\boldsymbol{\varphi}_{N}$ is the vector-valued counterpart of the function defined in equation (23). Again, the analogous structure of central scheme plus dissipation part is apparent.

This procedure can be extended to higher-order WENO schemes by only changing the function $\varphi_{N}$ of equation (27). For a $(2 r-1)$ WENO scheme, the central scheme will be of order $2(r-1)$, that is, one order less than the WENO scheme.

\section{Automatic Dissipation Adjustment (ADA) method}

The Automatic Dissipation Adjustment (ADA) method regulates the local dissipation adaptively based on some measurement of how well-resolved is the flow. This measurement is called the local energy ratio (ER) introduced in $[25,26]$. The ER can be seen as a ratio of the high-frequency velocity components using two different filters with different filtering widths.

$$
\mathrm{ER}=\frac{\sum\left(u_{i}-\widetilde{u}_{i}\right)^{2}}{\sum\left(u_{i}-\widehat{u}_{i}\right)^{2}}
$$

In equation (28), $u_{i}$ is the velocity field that comes from solving the incompressible Navier-Stokes equations, whereas $\hat{u}_{i}$ and $\tilde{u}_{i}$ are filtered velocities that result from applying filters with different widths. In this work, to ac-

count for the compressible effects, we follow [33] and we reformulate equation (28) as:

$$
\mathrm{ER}=\frac{\sum\left(\rho u_{i}-\widetilde{\rho u}_{i}\right)^{2}}{\sum\left(\rho u_{i}-\widehat{\rho u}_{i}\right)^{2}}
$$

In particular, the filters used to obtain $\widetilde{\rho u}_{i}$ and $\widehat{\rho u}_{i}$ are REV1: based on Moving Least Squares. In the following we briefly decribe the procedure 
to approximate a generic variable $\varphi$ by $\bar{\varphi}$ using $M L S$. The formulation, as derived in [34], yields

$$
\bar{\varphi}(\mathbf{x})=\mathbf{p}^{T}(\mathbf{x})\left[\mathbf{P}^{T}(\mathbf{x}) \mathbf{W}(\mathbf{x}) \mathbf{P}(\mathbf{x})\right]^{-1} \mathbf{P}^{T}(\mathbf{x}) \mathbf{W}(\mathbf{x}) \varphi(\mathbf{x})
$$

REV1: where $\mathbf{p}^{T}(\mathbf{x})=\left\{1, x, y, z, x y, x z, y z, x^{2}, \ldots\right\}$ is the selected polynomial basis, $\mathbf{P}^{T}(\mathbf{x})=\left[\mathbf{p}^{T}\left(\mathbf{x}_{j}\right)\right]_{j}$ gathers the evaluation of the basis functions in the points of the stencil, and $\mathbf{W}(\mathbf{x})=\operatorname{diag}\left(W_{j}\left(\left|\mathbf{x}-\mathbf{x}_{j}\right|, \kappa\right)\right)$ is the kernel matrix, that weights the relative importance of the points in the stencil. In this work, an exponential kernel is used, and its mathematical definition is given by

$$
W_{j}\left(\left|\mathbf{x}-\mathbf{x}_{j}\right|, \kappa\right)=\frac{\exp \left(-\left(\kappa \frac{\left|\mathbf{x}-\mathbf{x}_{j}\right|}{2 \max \left(\left|\mathbf{x}-\mathbf{x}_{j}\right|\right)}\right)^{2}\right)-\exp \left(-\kappa^{2}\right)}{1-\exp \left(-\kappa^{2}\right)} .
$$

REV1: We use two different different parameters $\kappa$ equal to 4 and 3 respectively as done in [33]. By varying the $\kappa$ parameter, different filtered fields are obtained. Subtracting them from the unfiltered field, yields an estimate of the high frequency content in the solution at that point. Note that the ER gives an indication about whether the flow is under-resolved or not. When there is an abundant high-frequency content, it means the existence of flow structures which are not well captured by the numerical scheme and the numerical grid. The energy at these scales cannot be correctly transferred to the smaller scales, and must be removed from the simulation in a way that mimics the procedure of the energy cascade. In order to do this, the proposed methodology decreases the numerical dissipation of the scheme.

\section{Mathematical formulation. WENO5 ADA scheme.}

The way of implementing the ADA mechanism into the WENO5 scheme is via a multiplicative coefficient $\varepsilon \in[0,1]$ that regulates the amount of dissipation introduced by the scheme. By adjusting the value of $\varepsilon$ dynamically at each time step and for every point in the grid, the scheme can adjust itself in a better fashion to the turbulent flow. The regions where the flow is well resolved, in the sense that the scales of the flow are properly captured by the numerical scheme and numerical grid, are going to have lower ER values compared to those where the flow is not well resolved. Thus, two threshold values 
are proposed in order to dissipate the excessive energy that accumulates on the smallest scales and to reduce the amount of dissipation on those locations where it is not needed. REV1: The threshold values have been selected after performing several numerical experiments. Note that these values have been adjusted for this numerical method and filters, and should remain unchanged for all the simulations. Note that the threshold values have been chosen to design a numerical scheme which is able to reproduce the Kolmogorov's law.

$$
\varepsilon_{i}^{n+1}=\left\{\begin{array}{cc}
\max \left[\left(\varepsilon_{i}^{n}-\phi\right), 0\right] & \mathrm{ER} \leq 1.50 \\
\min \left[\left(\varepsilon_{i}^{n}+\phi\right), 1\right] & \mathrm{ER} \geq 2.00 \\
\text { unmodified } & \text { case else }
\end{array} \mid \phi=0.05\right.
$$

where value of $\phi$ is selected to allow a smooth variation on the value of $\varepsilon$ over time. At first, the ADA mechanism can be thought to be implemented into the WENO5 scheme at two different stages. Namely, during the flux splitting or during the WENO reconstruction. The former approach, although seemingly valid at first glance, would imply a violation of the necessary condition explained in [31] where the flux splitting needs to have at least the same number of continuous derivatives as the order of the scheme. Thus, inserting a new $\varepsilon$ into the flux splitting procedure, as $\mathbf{F}_{i}^{ \pm}=\frac{1}{2}\left(\mathbf{F}_{i} \pm \varepsilon_{i+1 / 2} \alpha_{i} \mathbf{U}_{i}\right)$ introduces a discontinuity that rules out this possibility. This is clearly seen by supposing a constant flux $\mathbf{F}$, with different values of $\varepsilon$. The fluxes resulting from the splitting vary when the value of $\varepsilon$ varies, forming discontinuities and making the flux splitting of class $\mathcal{C}^{0}$.

The latter approach, introduces the $\varepsilon$ as a multiplicative coefficient to modify the dissipative part of the WENO scheme, without modifying the flux splitting procedure. This way, the condition on the derivative remains untouched and the dissipation can be adjusted according to the properties of the flow. Thus, the formulation would be modified as

$$
\begin{aligned}
\mathbf{F}_{i+1 / 2} & =\frac{1}{12}\left(-\mathbf{F}_{i-1}+7 \mathbf{F}_{i}+7 \mathbf{F}_{i+1}-\mathbf{F}_{i+2}\right) \\
& -\varepsilon_{i+1 / 2} \mathbf{R}_{i+1 / 2} \varphi_{N}\left(\Delta \mathcal{F}_{i-3 / 2}^{+}, \Delta \mathcal{F}_{i-1 / 2}^{+}, \Delta \mathcal{F}_{i+1 / 2}^{+}, \Delta \mathcal{F}_{i+3 / 2}^{+}\right) \\
& +\varepsilon_{i+1 / 2} \mathbf{R}_{i+1 / 2} \varphi_{N}\left(\Delta \mathcal{F}_{i+5 / 2}^{-}, \Delta \mathcal{F}_{i+3 / 2}^{-}, \Delta \mathcal{F}_{i+1 / 2}^{-}, \Delta \mathcal{F}_{i-1 / 2}^{-}\right)
\end{aligned}
$$

Since $\varepsilon_{i+1 / 2} \in[0,1]$, the scheme ranges from a central fourth-order Finite Difference scheme to a fully fifth-order WENO scheme. In order not to priv- 
ilege any sweeping direction over any other, we take $\varepsilon_{i+1 / 2}=\max \left(\varepsilon_{i}, \varepsilon_{i+1}\right)$. This way, we ensure that the reconstructed value is the same regardless of the sweeping direction (left to right or right to left).

This scheme may be unstable and may generate oscillations on regions with high gradients of pressure and/or density due to the fact that the original amount of dissipation is reduced. Thus, to design a robust and reliable scheme, an a posteriori approach will be used. This approach is based on the MOOD framework of [27, 35, 28, 36]. In [37] there is an extensive descriptions of the detectors that are commonly used in the REV2: Multidimensional Optimal Order Detection (MOOD) framework. In this work, REV2: we will only detail the ones employed in our formulation.

Physical Admissible Detector (PAD): This detector checks that all points in the solution have positive density and pressure at all times. In practice, it also accounts for points with NaN values.

Numerical Admissible Detector (NAD) [38]: relaxed version of the Discrete Maximum Principle (DMP)[27]. It checks that no new extrema are created and thus, the solution is monotonic. It compares the candidate solution with the solution obtained in the previous RungeKutta step.

$$
\begin{array}{r}
\min _{\mathbf{y} \in \mathcal{V}_{i}}\left(\mathbf{U}^{\mathrm{RK}}(\mathbf{y})\right)-\delta \leqslant \mathbf{U}^{*}(\mathbf{x}) \leqslant \max _{\mathbf{y} \in \mathcal{V}_{i}}\left(\mathbf{U}^{\mathrm{RK}}(\mathbf{y})\right)+\delta \\
\delta=\max \left(10^{-4}, 10^{-3} \cdot\left(\max _{\mathbf{y} \in \mathcal{V}_{i}}\left(\mathbf{U}^{\mathrm{RK}}(\mathbf{y})\right)-\min _{\mathbf{y} \in \mathcal{V}_{i}}\left(\mathbf{U}^{\mathrm{RK}}(\mathbf{y})\right)\right)\right)
\end{array}
$$

The collection of points $\mathcal{V}_{i}$ represents the set of first neighbors of the point in consideration. This implies that the candidate value remains between the local minimum and local maximum of the previous time step.

If the PAD and/or NAD are activated, we recalculate the interfaces values of the cell using $\varepsilon_{i+1 / 2}=1$, that is, forcing to use the full fifth-order WENO scheme. This way, a correct treatment of shock regions is guaranteed.REV2: In figure 2, the present approach is described in pseudocode. 


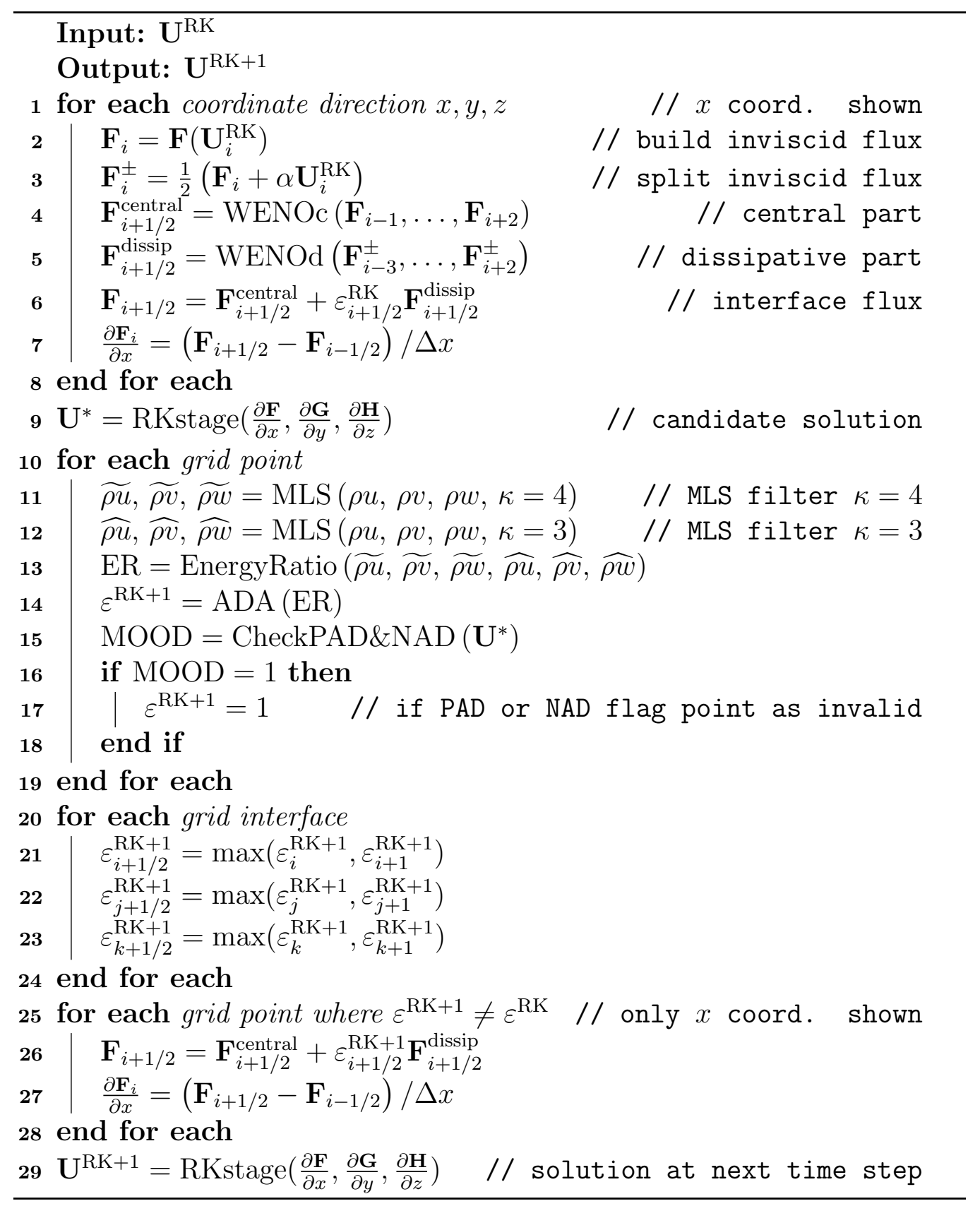

Figure 2: REV2: Pseudocode algorithm of the WENO5 ADA procedure. For brevity, the reconstruction of the viscous fluxes is omitted. 


\section{Discretization of the viscous fluxes}

REV2: The proposed methodology only applies to the inviscid part of the Navier-Stokes equations. The viscous fluxes will be treated as in [39], which is briefly described in the following. The main advantage of this procedure is that it uses the same WENO5 stencil, thus there is no need of using additional ghost points. The derivative of a generic viscous flux $R$ along the $x$ coordinate (same applies for $y$ and $z$ directions) is computed as

$$
\left.\frac{\partial R}{\partial x}\right|_{i j k}=\hat{R}_{i+1 / 2, j, k}-\hat{R}_{i-1 / 2, j, k} .
$$

REV2: where $\hat{R}_{i-1 / 2, j, k}$ is computed with at least fourth order of accuracy as

$$
\hat{R}_{i-1 / 2, j, k}=\frac{1}{24 \Delta x}\left(-R_{i+1 / 2, j, k}+26 R_{i-1 / 2, j, k}-R_{i-3 / 2, j, k}\right) .
$$

REV2: All entries of the viscous flux vectors are interpolated to the corresponding set of interfaces $I=\{i-3 / 2, i-1 / 2, i+1 / 2\}$ using the following formulas

$$
\begin{aligned}
u_{I} & =\sum_{l=-2}^{1} C_{l}^{I} u_{i+l, j, k} \\
\left.\frac{\partial u}{\partial \xi}\right|_{I} & =\frac{1}{\Delta \xi} \sum_{l=-3}^{2} D_{l}^{I} u_{i+l, j, k} \\
\left.\frac{\partial u}{\partial \eta}\right|_{I} & =\left.\sum_{l=-2}^{1} C_{l}^{I} \frac{\partial u}{\partial \eta}\right|_{i+l, j, k} \\
\left.\frac{\partial u}{\partial \eta}\right|_{i, j, k} & =\frac{1}{\Delta \eta} \sum_{l=-2}^{2} C_{l}^{c} u_{i, j+l, k}
\end{aligned}
$$

REV2: Equation (38) is used to interpolate the variables' values at the interfaces, equation (39) is used to interpolate the derivative along the corresponding direction, whereas equations (40) and (41) are used for interpolating derivatives along any traversal direction. The coefficients for the interpolations can be seen in tables 1 to 3. 


\begin{tabular}{ccccc}
\hline$I$ & $C_{-2}^{I}$ & $C_{-1}^{I}$ & $C_{0}^{I}$ & $C_{1}^{I}$ \\
\hline$i-3 / 2$ & $5 / 16$ & $15 / 16$ & $-5 / 16$ & $1 / 16$ \\
$i-1 / 2$ & $-1 / 16$ & $9 / 16$ & $9 / 16$ & $-1 / 16$ \\
$i+1 / 2$ & $1 / 16$ & $-5 / 16$ & $15 / 16$ & $5 / 16$ \\
\hline
\end{tabular}

Table 1: The coefficients $C_{l}^{I}$ taken from [39]

\begin{tabular}{ccccccc}
\hline $\mathrm{I}$ & $D_{-3}^{I}$ & $D_{-2}^{I}$ & $D_{-1}^{I}$ & $D_{0}^{I}$ & $D_{1}^{I}$ & $D_{2}^{I}$ \\
\hline$i-3 / 2$ & $71 / 1920$ & $-141 / 128$ & $69 / 64$ & $1 / 192$ & $-3 / 128$ & $3 / 640$ \\
$i-1 / 2$ & $-3 / 640$ & $25 / 384$ & $-75 / 64$ & $75 / 64$ & $-25 / 384$ & $3 / 640$ \\
$i+1 / 2$ & $-3 / 640$ & $3 / 128$ & $-1 / 192$ & $-69 / 64$ & $141 / 128$ & $-71 / 1920$ \\
\hline
\end{tabular}

Table 2: The coefficients $D_{l}^{I}$ taken from [39]

\begin{tabular}{ccccc}
\hline$C_{-2}^{c}$ & $C_{-1}^{c}$ & $C_{0}^{c}$ & $C_{1}^{c}$ & $C_{2}^{c}$ \\
\hline $1 / 12$ & $-8 / 12$ & 0 & $8 / 12$ & $-1 / 12$ \\
\hline
\end{tabular}

Table 3: The coefficients $C_{l}^{c}$ taken from [39]

\section{Numerical examples}

In the following section different one- and three-dimensional problems will be tested in order to assess the proposed WENO5 ADA scheme. All the simulations are run using the explicit TVDRK3 of [3] as time integration scheme, and $\gamma=1.4$ unless otherwise noted.

\subsection{One-dimensional test cases}

In order to validate the proposed scheme, several one-dimensional test cases will be analyzed with the WENO5 ADA and compared to the results obtained by the classical WENO5M scheme. The focus is placed on inviscid cases, since the ADA mechanism modifies the convective part of the equations. 


\subsubsection{Double shock test case}

This test case involves two shocks moving away from each other. The initial data is a slight variation of the one that can be found in [40] and can be expressed as:

$$
(\rho, u, p)=\left\{\begin{array}{ll}
\left(\rho_{L}, u_{L}, p_{L}\right) & \text { if } \quad x \leq 0.5 \\
\left(\rho_{R}, u_{R}, p_{R}\right) & \text { if } \quad x>0.5
\end{array} \text { where } \begin{array}{l}
\left(\rho_{L}, u_{L}, p_{L}\right)=(6.0,20.0,250.0) \\
\left(\rho_{R}, u_{R}, p_{R}\right)=(6.0,-6.0,45.0)
\end{array}\right.
$$

The simulation will be run until the final time of $t=0.02$ and the CFL will be held constant at a value of 0.5 . Neumann boundary conditions are applied on both ends of the domain $[0,1]$.
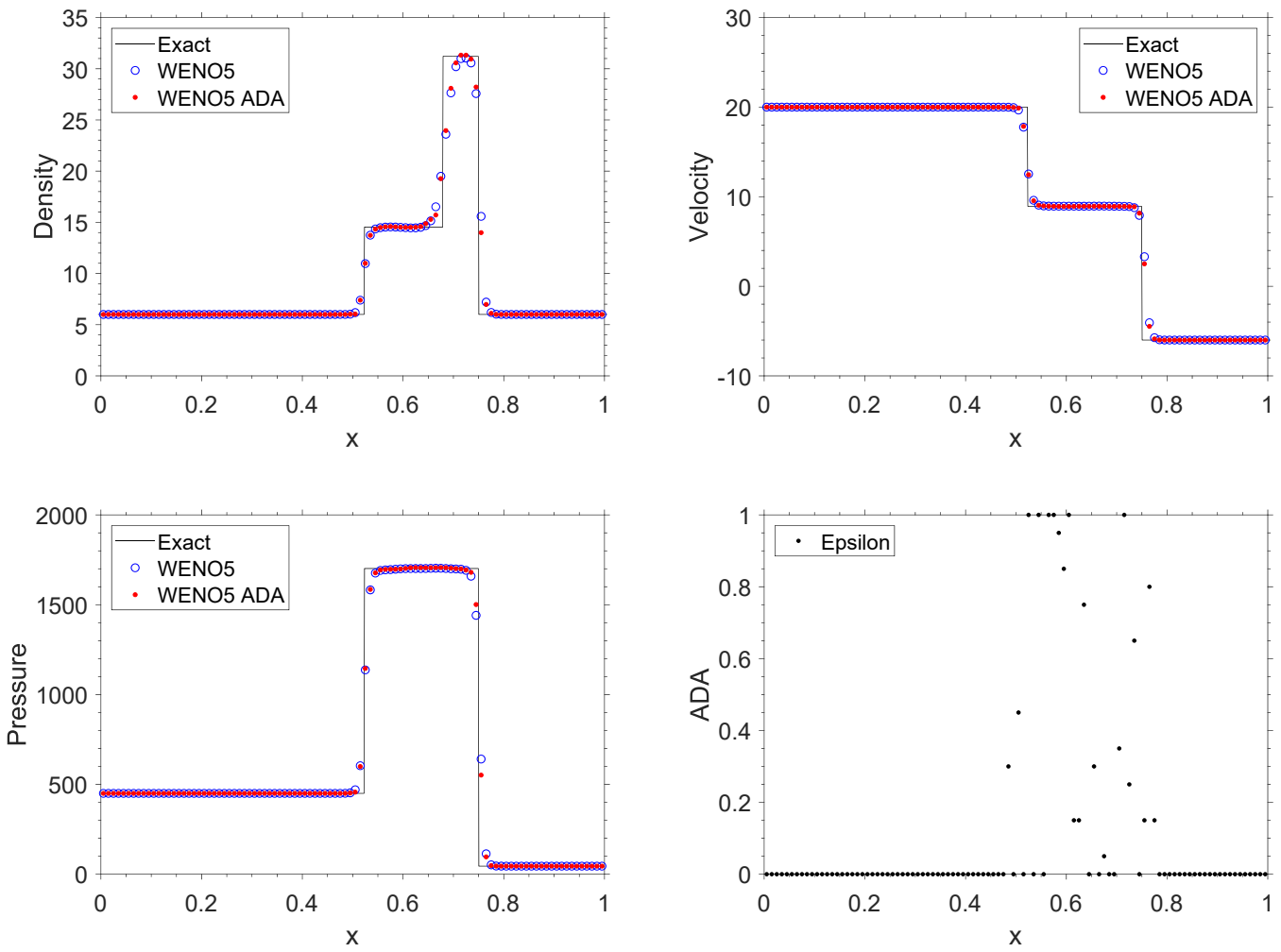

Figure 3: Double shock test case. Obtained results for a 100-point grid at the final time of $t=0.02$.

Figure 3 shows the results for the WENO5 and WENO5 ADA schemes. A very similar solution is obtained with both schemes, while the latter has better performance around discontinuities. No numerical issues are observed on 
the performance of the WENO ADA scheme such as dispersion or dissipation errors.

\subsubsection{Left expansion and right strong shock test case}

This test case involves a right-moving shock and a left-moving rarefaction. The initial data is a slight variation of the ones that can be found in [40] and can be expressed as:

$$
(\rho, u, p)=\left\{\begin{array}{cc}
\left(\rho_{L}, u_{L}, p_{L}\right) & \text { if } x \leq 0.5 \\
\left(\rho_{R}, u_{R}, p_{R}\right) & \text { if } x>0.5
\end{array} \text { with } \begin{array}{l}
\left(\rho_{L}, u_{L}, p_{L}\right)=(3.0,0.0,1000.0) \\
\left(\rho_{R}, u_{R}, p_{R}\right)=(2.0,0.0,0.1)
\end{array}\right.
$$

The simulation will be run until the final time of $t=0.02$ with a CFL value of 0.5 . Neumann boundary conditions are applied on both ends of the domain $[0,1]$.
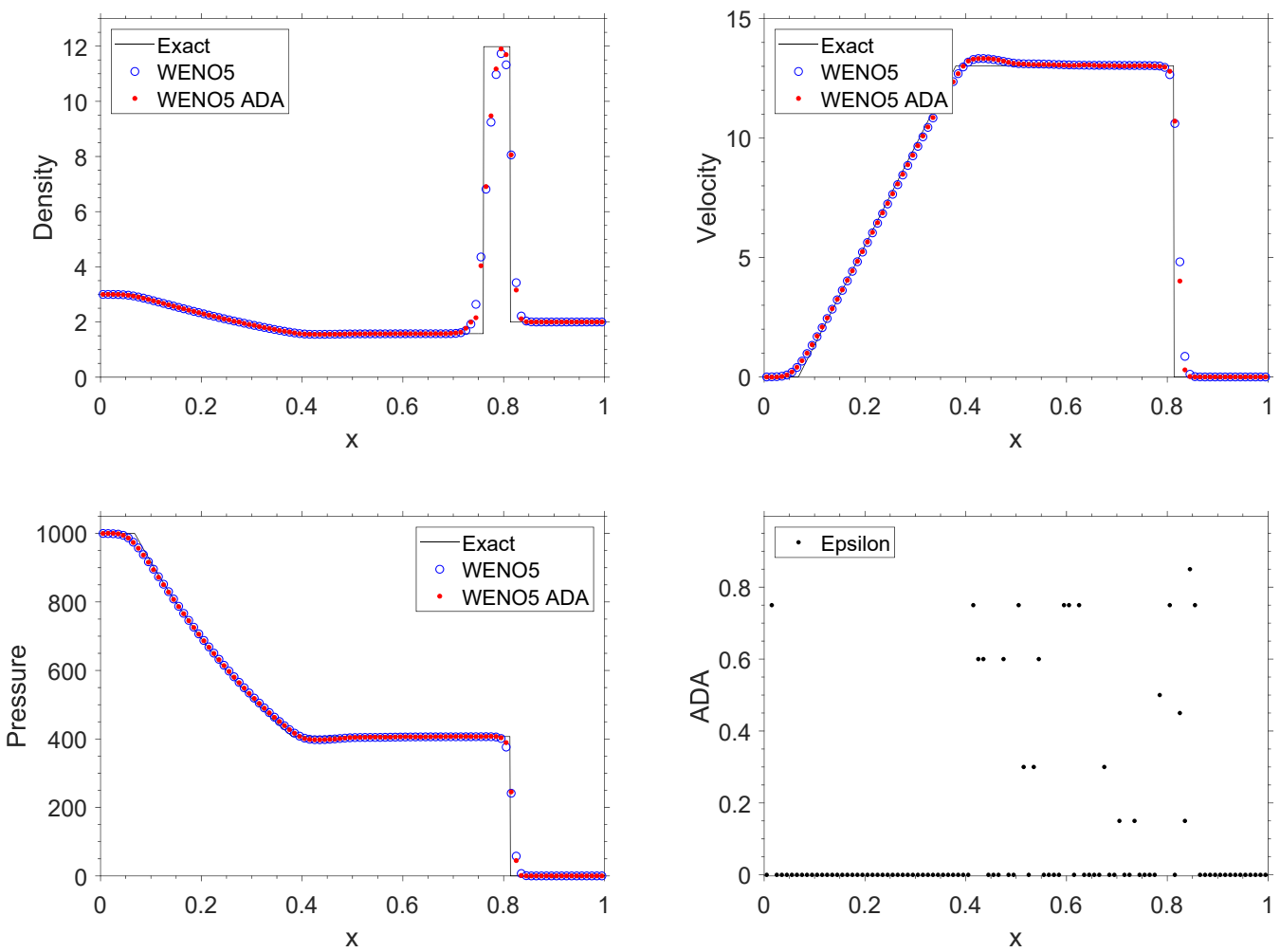

Figure 4: Left expansion and right strong shock test case. Obtained results for a 100-point grid at the final time of $t=0.02$. 
From Figure 4, similar conclusions to those of the previous test case can be drawn for the WENO5 and WENO5 ADA schemes.

\subsubsection{Shock/Density oscillation interaction test case}

This one-dimensional test case, proposed in [30], is a Mach 3 shock wave interacting with upstream sinusoidal density waves. The domain is defined in $-5 \leq x \leq 5$ and the boundary conditions are set as inflow at $x=-5$ and outflow at $x=5$. The solution is computed with a CFL number equal to 0.5 and the final time of the simulation is $t=1.8$. The non-dimensional initial condition is defined as:

$$
(\rho, u, p)=\left\{\begin{array}{ccc}
(3.857143,2.629369,10.33333) & \text { if } \quad x<-4 \\
(1+0.2 \sin (5 x), 0,1) & \text { if } \quad x \geq-4
\end{array}\right.
$$
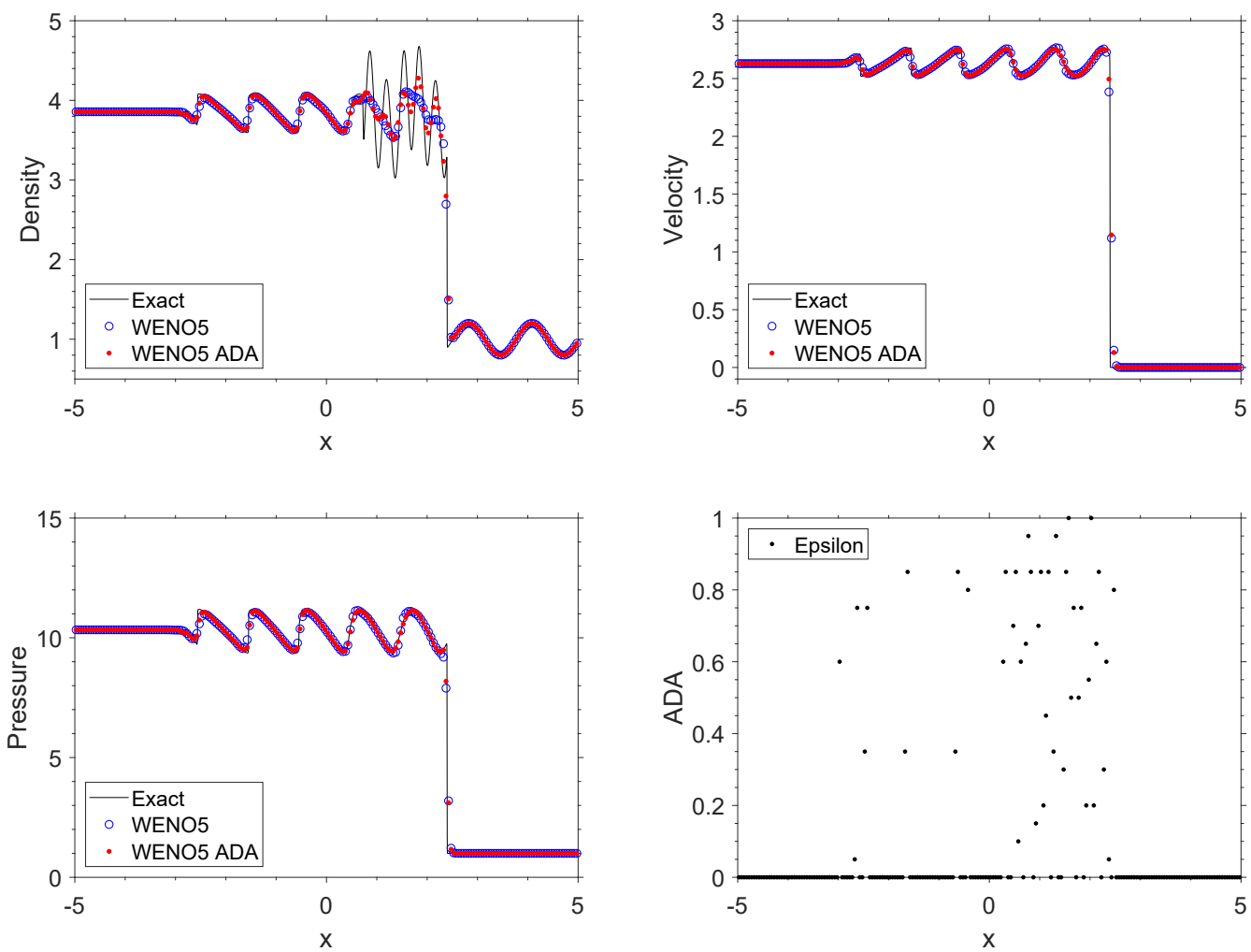

Figure 5: Shock/Density oscillation interaction test case. Obtained results for a REV3: 200-point grid at the final time of $t=1.8$. Density (top left), Velocity (top right), Pressure (bottom left) and value of epsilon for the WENO5 ADA scheme (bottom right). 
This test measures the ability of the schemes for capturing both smallscale smooth flow and shocks. It is a common benchmark in the context of turbulence solvers. The overall performance of the WENO5 ADA scheme can be seen in figure 5 and with more detail for the density plot in figure 6 . Since this test case is designed to be a turbulence benchmark, an important improvement can be observed in the oscillatory region, where the WENO5 ADA scheme performs better than its original counterpart.

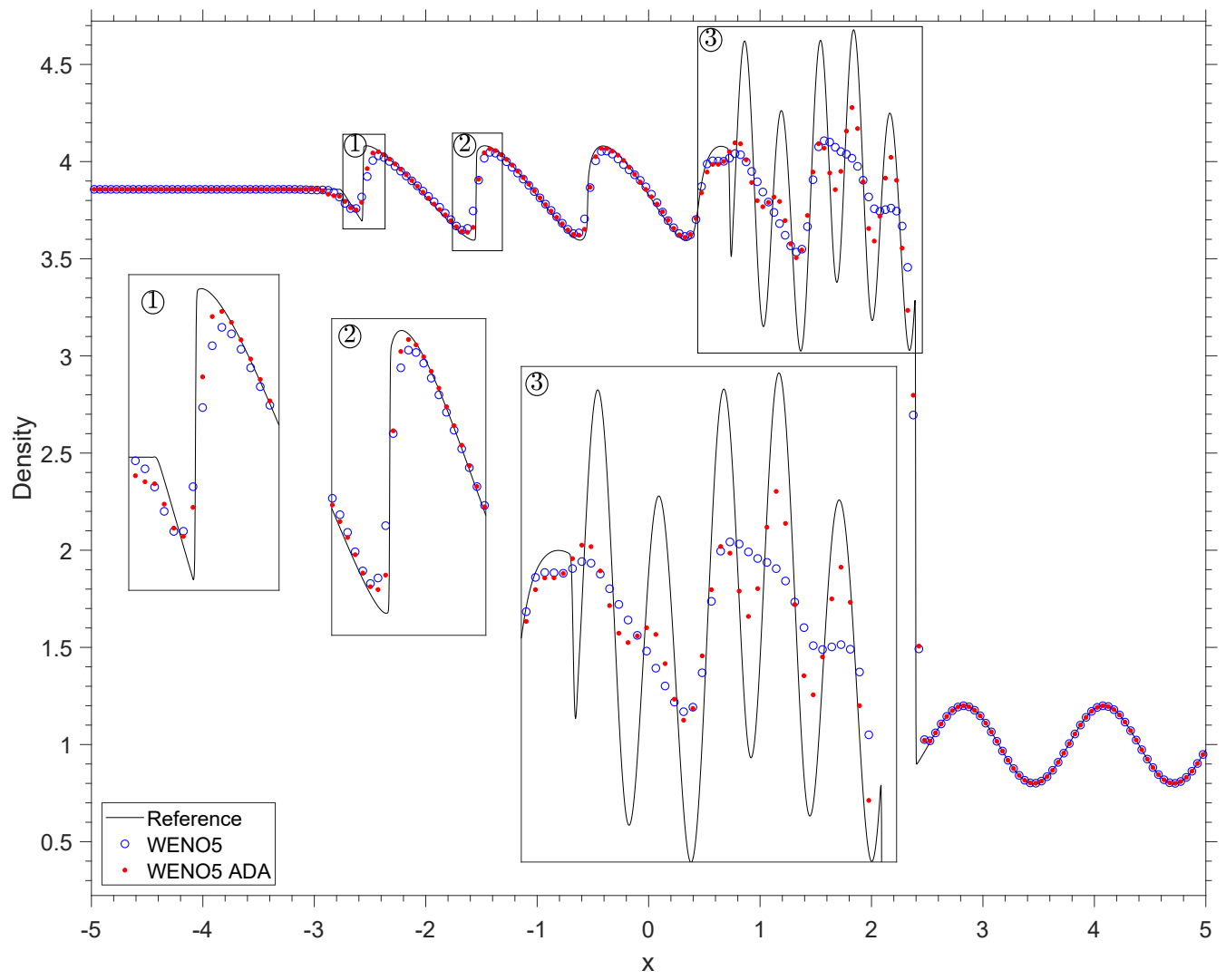

Figure 6: Shock/Density oscillation interaction test case. Obtained results for a REV3: 200-point grid at the final time of $t=1$.8. Density details.

As seen in the presented one-dimensional test cases, the ADA mechanism delivers a really similar solution to that of the WENO5 scheme, achieving a sharper resolution around contact discontinuities and shocks. There are no phase or dissipation errors in the solutions obtained with the WENO5 ADA method and, thus, REV3: two-dimensional and three-dimensional test cases 
will be studied on the following sections.

\subsection{Two-dimensional test cases}

\subsubsection{Entropy Wave 2D}

REV1: In order to assess the accuracy of the proposed scheme, we perform a mesh refinement study using the advection of a two-dimensional entropy wave with periodic boundary conditions. This test case can be consulted in [41] and takes place in a 2-by-2 square domain. The time evolution of the wave can be expressed as

$$
\begin{aligned}
& \rho(x, y, t)=\rho_{\infty}+A \sin \left[\pi\left(x+y-\left(u_{\infty}+v_{\infty}\right) t\right)\right] \\
& u(x, y, t)=u_{\infty} \\
& v(x, y, t)=v_{\infty} \\
& p(x, y, t)=p_{\infty}
\end{aligned}
$$

REV1: where $A=0.2, \rho_{\infty}=1, u_{\infty}=1, v_{\infty}=1$ and $p_{\infty}=1$. The simulation is carried out until $t=2$, thus completing a full revolution around the domain. In order to measure the accuracy, we compare the solution at the end of the simulation with the analytic one. We obtain the $L_{1}, L_{2}$ and $L_{\infty}$ error norms, defined respectively as

$$
L_{1}(\phi)=\frac{1}{|\Omega|} \sum_{k \in \Omega}\left|\phi_{k}\right|, \quad L_{2}(\phi)=\sqrt{\frac{1}{|\Omega|} \sum_{k \in \Omega}\left|\phi_{k}\right|^{2}}, \quad L_{\infty}(\phi)=\max _{k \in \Omega}\left|\phi_{k}\right|
$$

\begin{tabular}{cccccccc}
\hline \multicolumn{8}{c}{ WENO5 } \\
\hline Mesh & $L_{1}$ & $r_{1}$ & $L_{2}$ & $r_{2}$ & $L_{\infty}$ & $r_{\infty}$ & Time $(\mathrm{s})$ \\
\hline $25 \times 25$ & $2.205 \mathrm{E}-05$ & - & $3.014 \mathrm{E}-05$ & - & $9.384 \mathrm{E}-05$ & - & 4.23 \\
\hline $50 \times 50$ & $6.756 \mathrm{E}-07$ & 5.03 & $9.254 \mathrm{E}-07$ & 5.03 & $2.979 \mathrm{E}-06$ & 4.98 & 19.87 \\
\hline $100 \times 100$ & $2.152 \mathrm{E}-08$ & 4.98 & $2.937 \mathrm{E}-08$ & 4.98 & $9.636 \mathrm{E}-08$ & 4.95 & 127.60 \\
\hline $200 \times 200$ & $7.484 \mathrm{E}-10$ & 4.86 & $1.010 \mathrm{E}-09$ & 4.86 & $3.518 \mathrm{E}-09$ & 4.78 & 1192.47 \\
\hline
\end{tabular}

Table 4: Entropy Wave 2D. WENO5 order convergence results. 


\begin{tabular}{cccccccc}
\hline \multicolumn{8}{c}{ WENO5 ADA } \\
\hline Mesh & $L_{1}$ & $r_{1}$ & $L_{2}$ & $r_{2}$ & $L_{\infty}$ & $r_{\infty}$ & Time (s) \\
\hline $25 \times 25$ & $6.885 \mathrm{E}-05$ & - & $9.812 \mathrm{E}-05$ & - & $5.024 \mathrm{E}-04$ & - & 5.61 \\
\hline $50 \times 50$ & $4.809 \mathrm{E}-06$ & 3.84 & $6.410 \mathrm{E}-06$ & 3.94 & $2.739 \mathrm{E}-05$ & 4.20 & 26.53 \\
\hline $100 \times 100$ & $3.043 \mathrm{E}-07$ & 3.98 & $4.037 \mathrm{E}-07$ & 3.99 & $1.893 \mathrm{E}-06$ & 3.85 & 175.15 \\
\hline $200 \times 200$ & $1.913 \mathrm{E}-08$ & 3.99 & $2.533 \mathrm{E}-08$ & 3.99 & $1.247 \mathrm{E}-07$ & 3.92 & 1312.93 \\
\hline
\end{tabular}

Table 5: Entropy Wave 2D. WENO5 ADA order convergence results.

REV1: It can be seen in tables 4 and 5 that for smooth test cases like this one, the WENO5 ADA behaves as the fourth-order central finite difference scheme, since the NAD does not activate after the first few iterations and $\varepsilon=0$ thereafter. This effect has some impact in the overall accuracy of the proposed scheme, but remaining at least fourth-order at all times. In real test cases, the expected accuracy will vary between fourth-and fifth-order depending on the flow characteristics.

\subsubsection{D Riemann problem}

REV3: The two-dimensional Riemann problems constitute a family of different configurations that take place in a unitary square divided into four quadrants. Each quadrant has a different initial configuration. Among all the possible test cases, we chose case 12 of [42], whose initial setup is described in the equation (47).

$$
\frac{\left(\begin{array}{l}
\rho_{2} \\
u_{2} \\
v_{2} \\
p_{2}
\end{array}\right)=\left(\begin{array}{l}
1.0000 \\
0.7276 \\
0.0000 \\
1.0000
\end{array}\right) \mid\left(\begin{array}{l}
\rho_{1} \\
u_{1} \\
v_{1} \\
p_{1}
\end{array}\right)=\left(\begin{array}{l}
0.5313 \\
0.0000 \\
0.0000 \\
0.4000
\end{array}\right)}{\left(\begin{array}{l}
\rho_{3} \\
u_{3} \\
v_{3} \\
p_{3}
\end{array}\right)=\left(\begin{array}{l}
\rho_{4} \\
u_{4} \\
v_{4} \\
p_{4}
\end{array}\right)=\left(\begin{array}{l}
1.0000 \\
0.0000 \\
0.7276 \\
1.000000
\end{array}\right)}
$$

REV3: The initial condition for this test case presents two shocks and two contact discontinuities and the solution evolves while conserving the sym- 
metry around the $y=x$ line until the final time of the simulation $t=0.25$.

REV3: In figures 7 and 8, the WENO5 versus WENO5 ADA results are shown. The proposed approach is stable, accurately captures contact discontinuities (as opposed to its original counterpart that tends to spread them), at the price of allowing certain oscillations near the shock regions. These oscillations do not compromise the stability of the overall scheme, as mentioned, due to the a posterior detection method that was incorporated.

REV3: The detection for the final instant is shown in figure 9, and it is seen that the detection is gathered around the shock regions with small occurrences in other parts of the domain, as expected. This is more apparent in the finer grid.
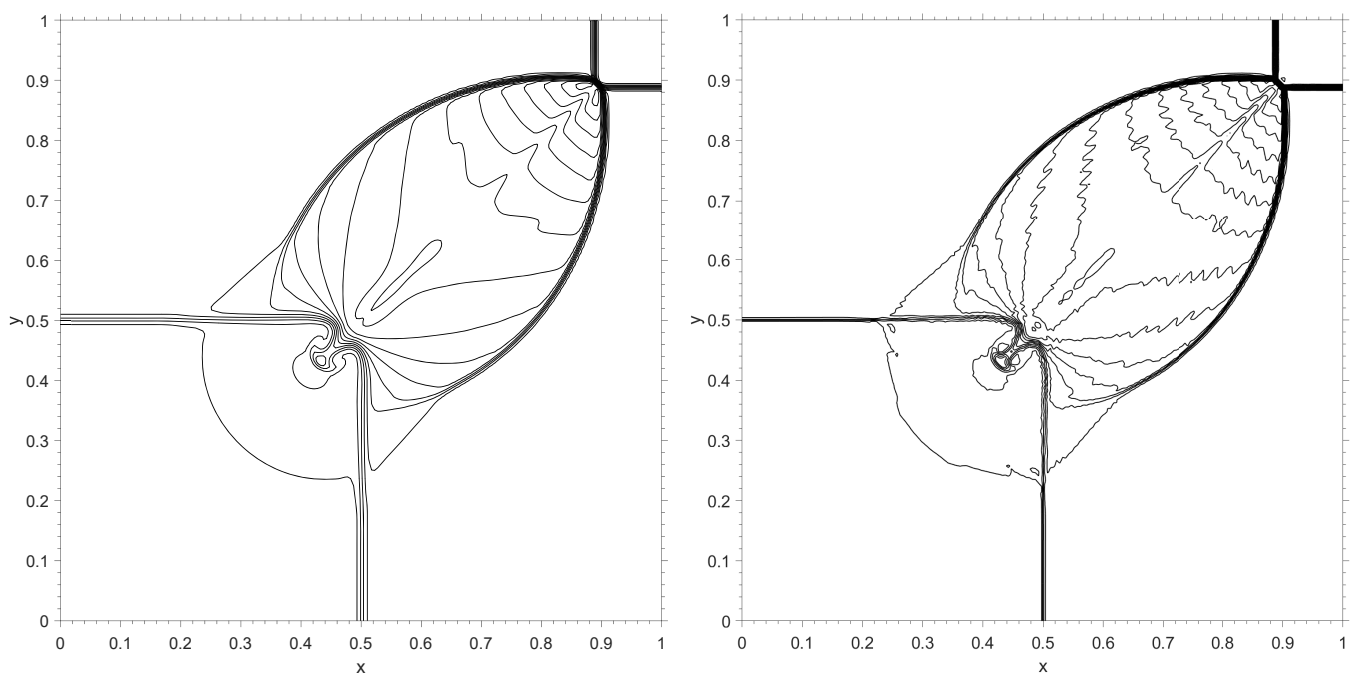

Figure 7: 2D Riemann problem. WENO5 (left) vs. WENO5 ADA (right). 25 equally spaced density contours from 0.53 to 1.72 at $t=0.25$. 200x200 mesh. 

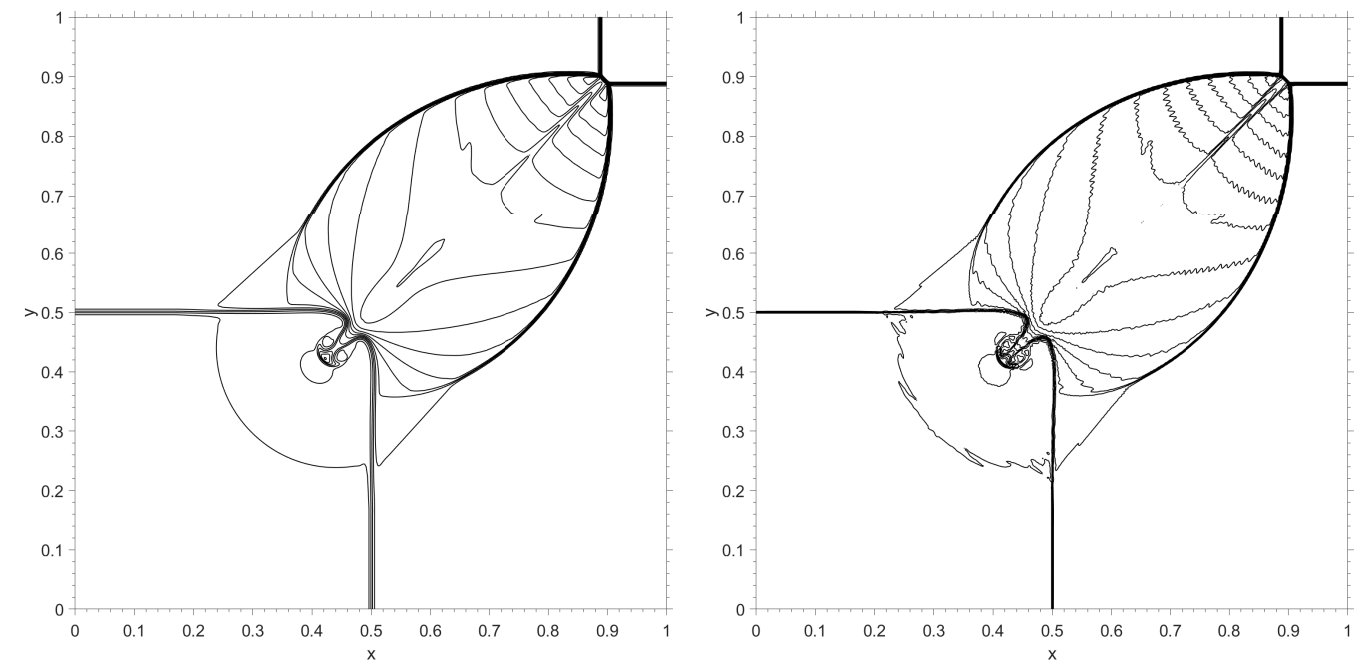

Figure 8: 2D Riemann problem. WENO5 (left) vs. WENO5 ADA (right). 25 equally spaced density contours from 0.53 to 1.72 at $t=0.25$. 400x400 mesh.
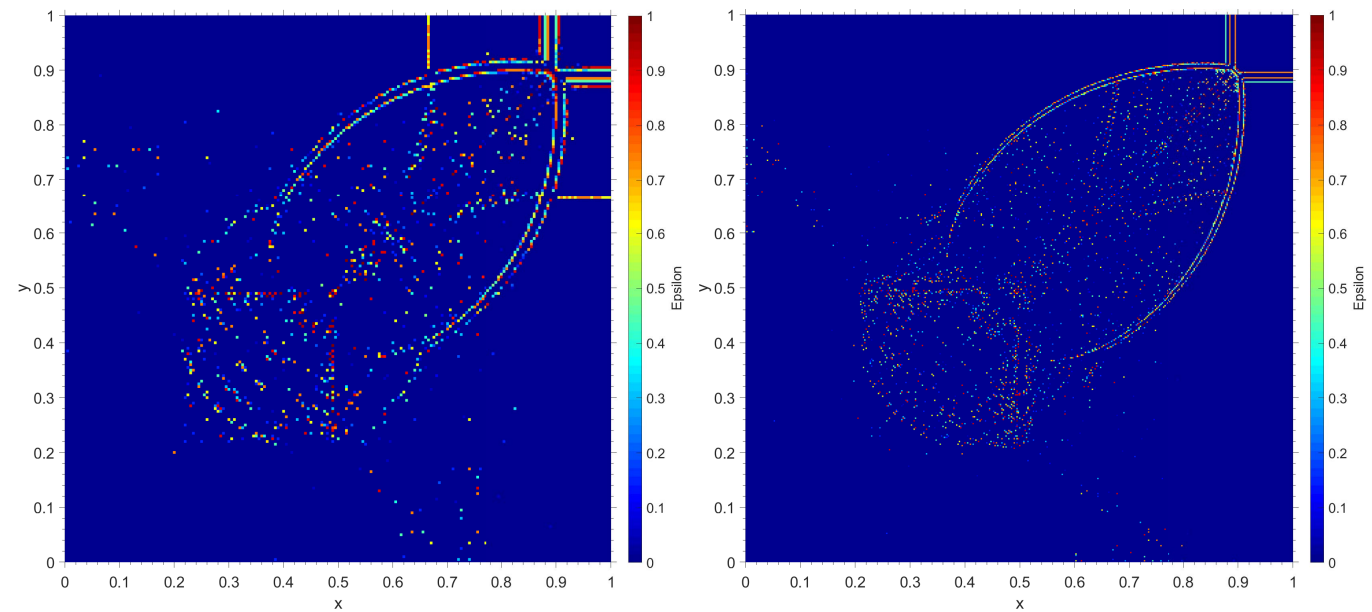

Figure 9: 2D Riemann problem. WENO5 ADA epsilon value distribution at $t=0.2$. 200x200 mesh (left) and 400x400 mesh (right). 


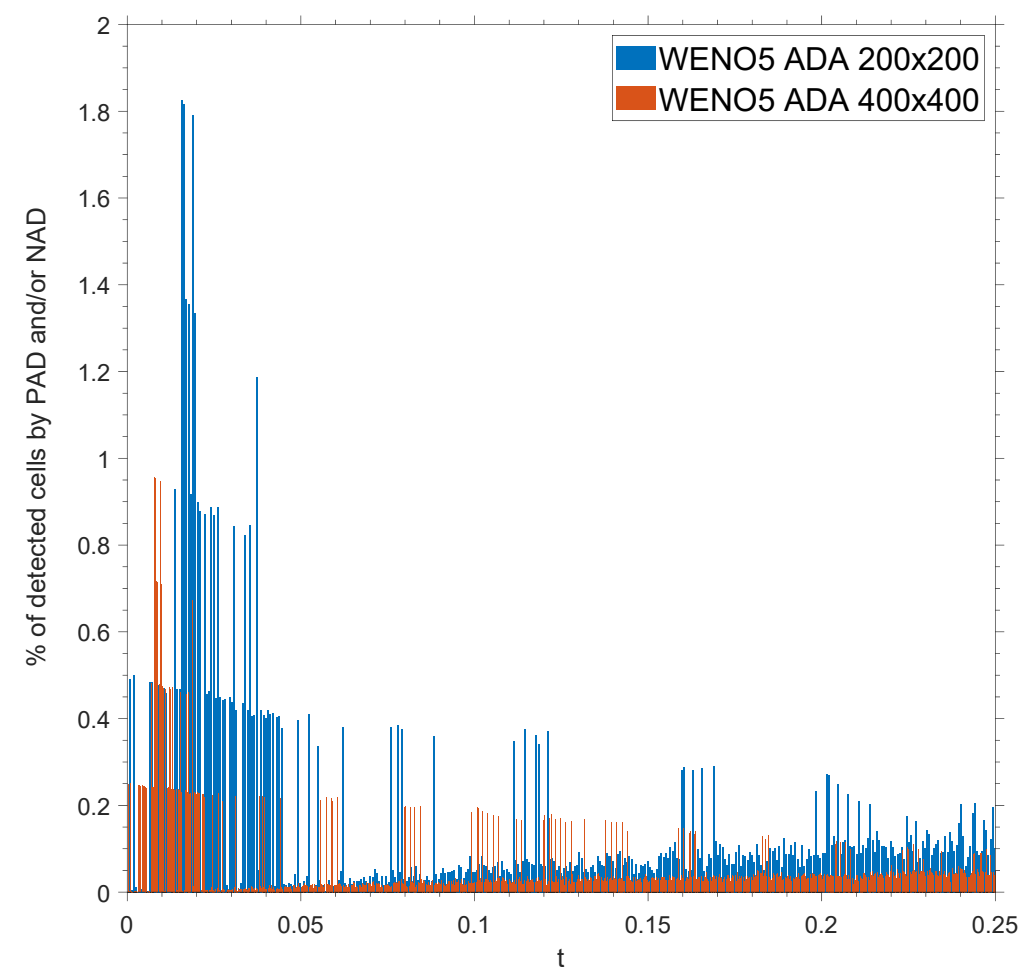

Figure 10: 2D Riemann problem. WENO5 ADA \% of detected cells during the simulation. 200x200 and 400x400 meshes.

REV3: The evolution of the detected cells along the simulation is shown in figure 10. For this test case, the troubled cells constitute a fraction of the total domain that is under $0.5 \%$, after the first few iterations have passed.

\subsubsection{Double Mach reflection}

REV3: This two-dimensional test case is used as a benchmark for Euler codes to assess their performance in the presence of strong discontinuities [43]. A Mach 10 shock reflects from an inviscid wall, resulting in secondary shock waves and contact discontinuities. It takes place in a $[0,4] \times[0,1]$ rectangular domain. The initial condition is an oblique Mach 10 shock that forms 60 degrees with the $x$ axis, intersecting the lower boundary at $x=1 / 6$.

REV3: The flow conditions upstream and downstream of the shock are respectively

$$
\begin{aligned}
& \left(\rho_{u}, u_{u}, v_{u}, p_{u}\right)=(8.000,7.145,-4.125,116.500) \\
& \left(\rho_{d}, u_{d}, v_{d}, p_{d}\right)=(1.400,0.000,0.000,1.000) .
\end{aligned}
$$


REV3: The bottom boundary $(y=0)$ is divided into two different regions: the first one $(0<x<1 / 6)$ takes the upstream flow conditions, while the second one $(x \geq 1 / 6)$ is an inviscid wall. The upper boundary $(y=1)$ takes the analytic values of a Mach 10 oblique shock. The left and right boundaries take up- and downstream flow conditions respectively. The simulation is run until $t=0.2$.

REV3: In figures 11 and 12, the WENO5 versus WENO5 ADA results are shown. The proposed approach, once again, is stable, and obtains really similar results to the WENO5

REV3: The detection for the final instant is shown in figure 13, and it is seen that it is gathered around the shock regions but also in the inner area between the shoch and the bottom wall. This behavior is explained because a Mach 10 shock is really strong and the initial shock going into the wall generates oscillations that trigger the NAD detector in that region.
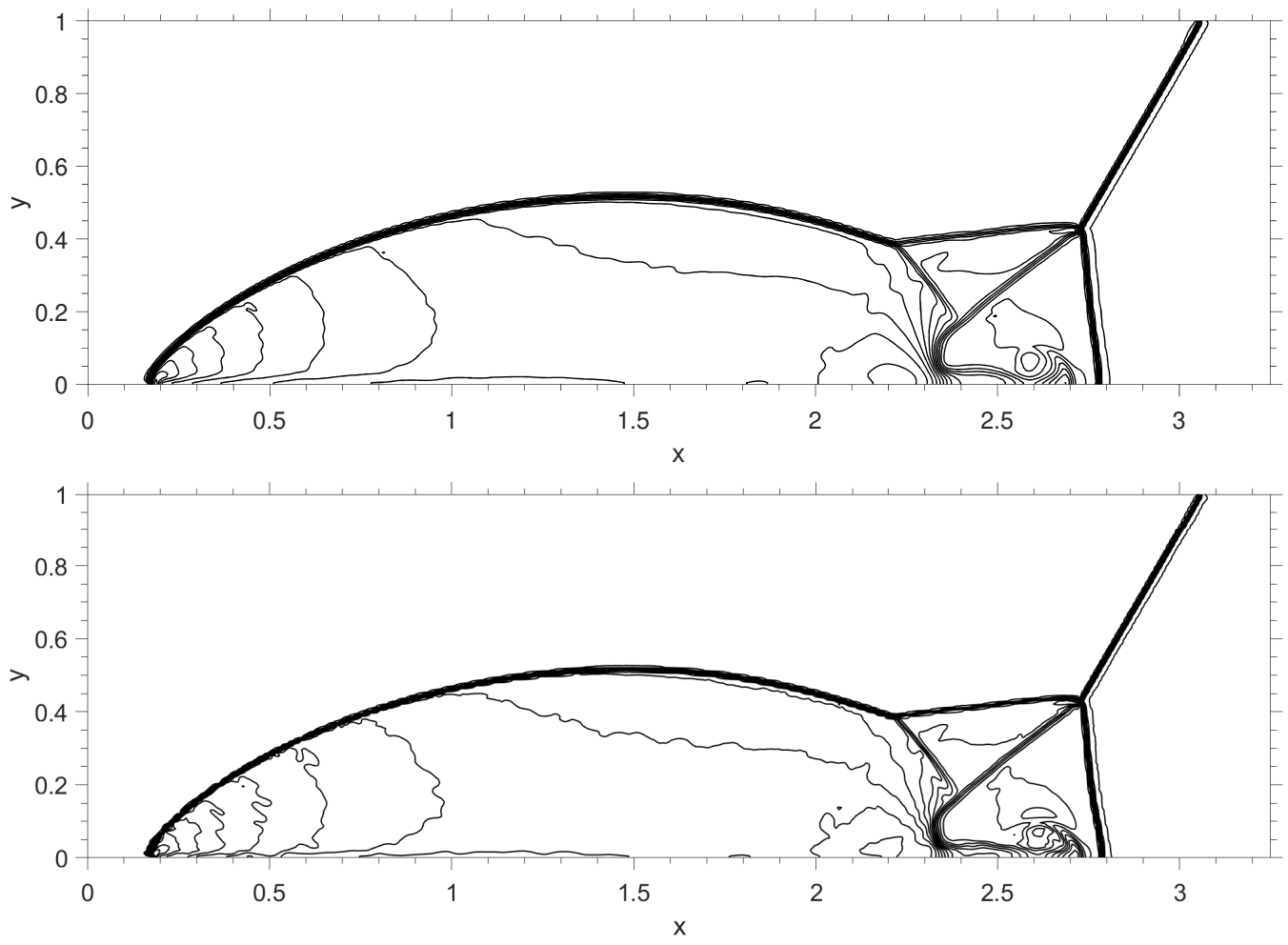

Figure 11: Double Mach reflection. WENO5 (top) vs. WENO5 ADA (bottom). 30 equally spaced density contours from 1.4 to 21.6 at $t=0.2$. $480 \mathrm{x} 120$ mesh. 

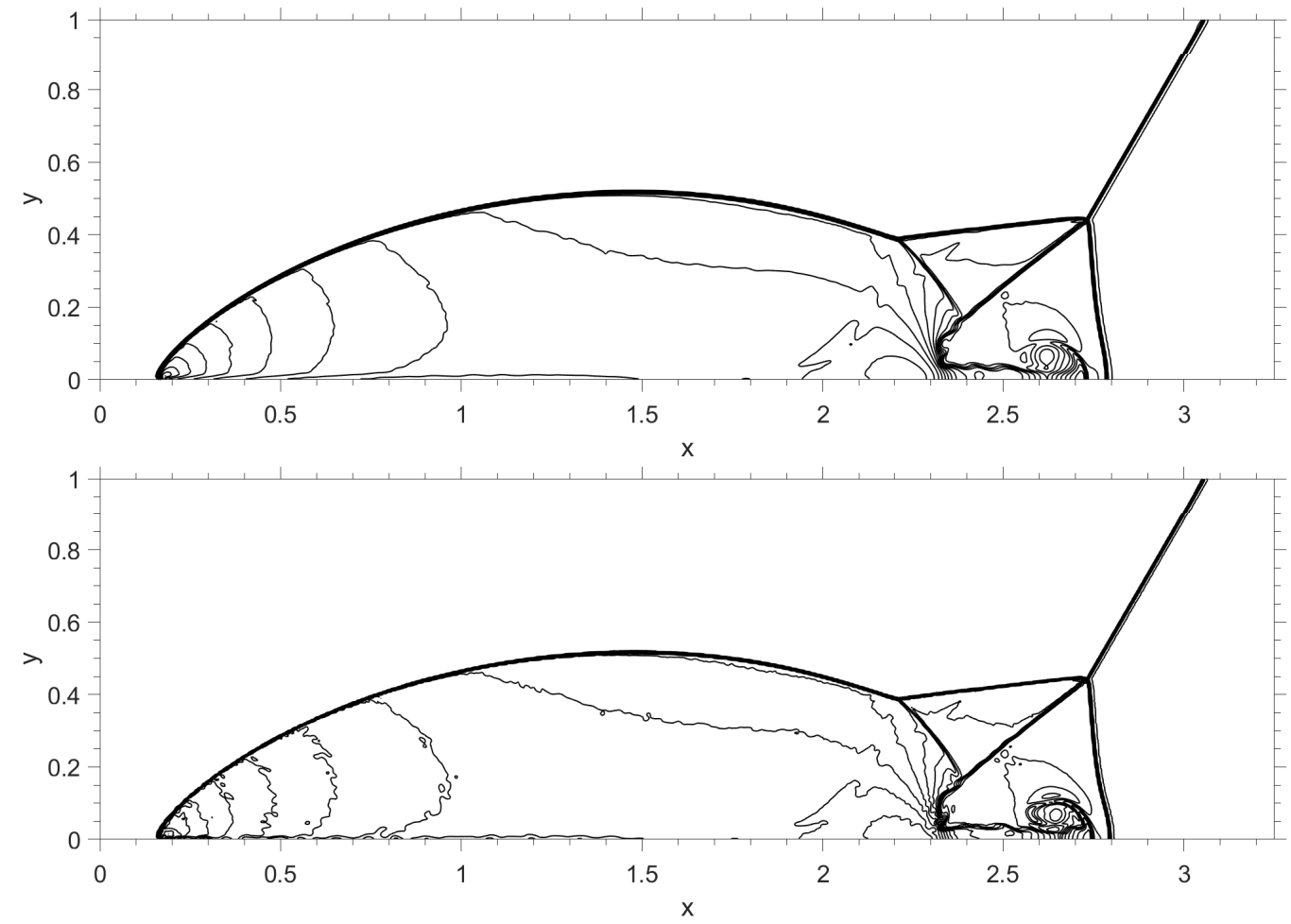

Figure 12: Double Mach reflection. WENO5 (top) vs. WENO5 ADA (bottom). 30 equally spaced density contours from 1.4 to 21.6 at $t=0.2$. 960x240 mesh. 

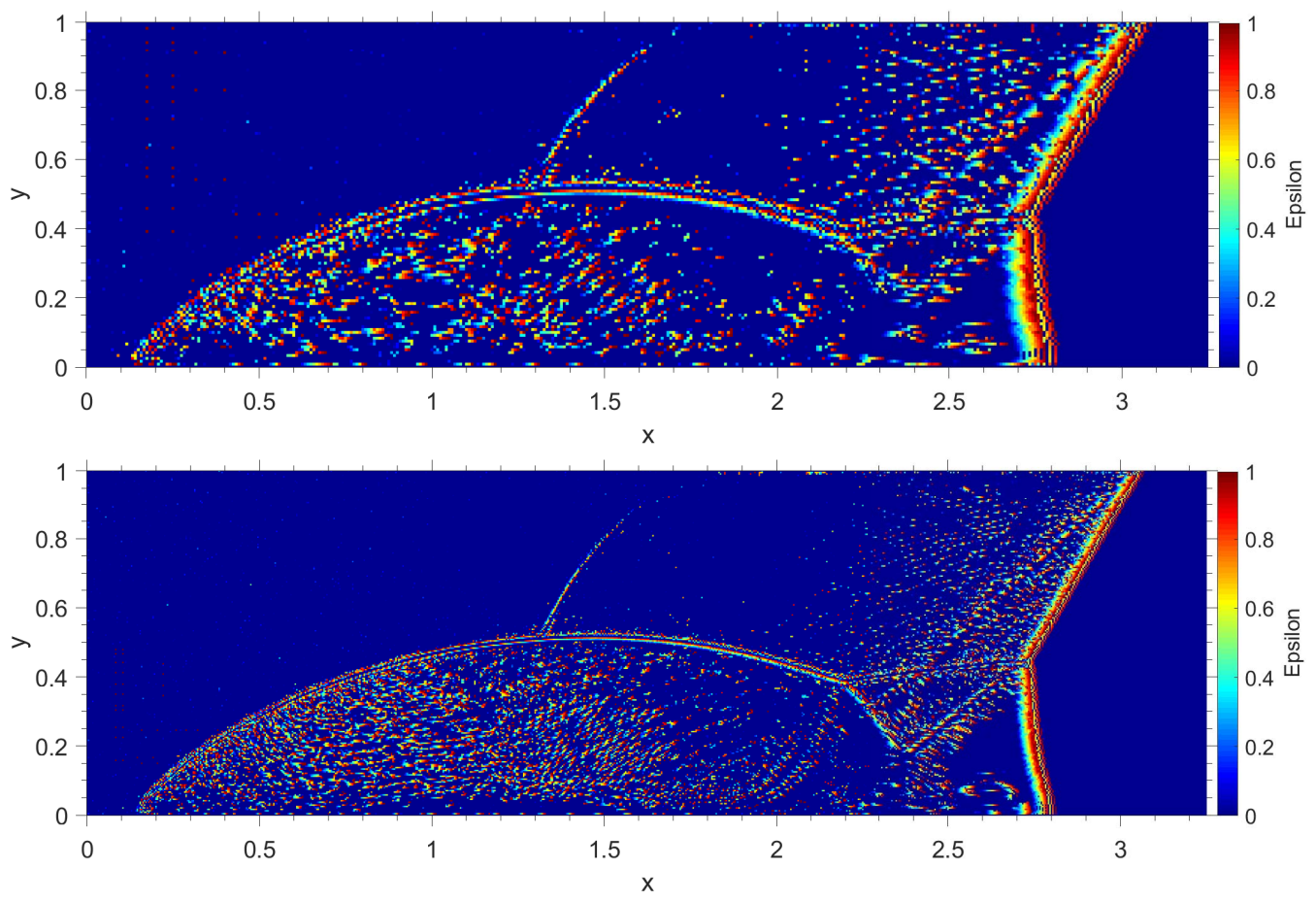

Figure 13: Double Mach reflection. WENO5 ADA epsilon value distribution at $t=0.2$. 480x120 (top) and 960x240 (bottom) meshes. 


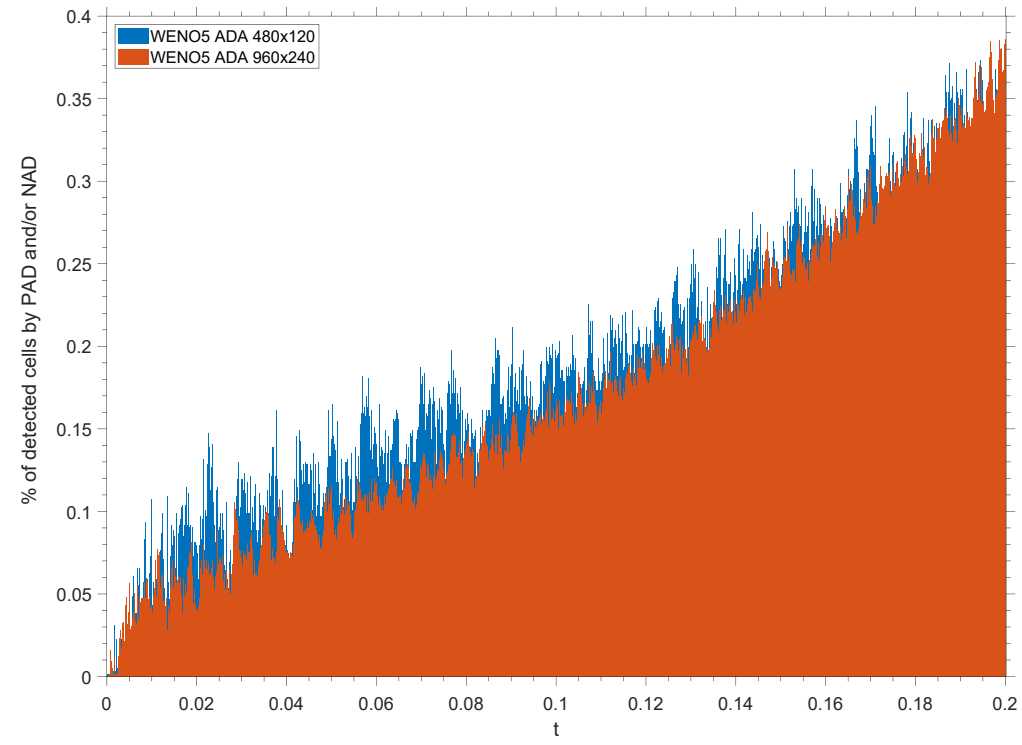

Figure 14: Double Mach reflection. WENO5 ADA \% of detected cells during the simulation. 480x120 and 960x240 meshes.

REV3: The evolution of the detected cells along the simulation is shown in figure 14. For this test case, the troubled cells constitute a fraction of the total domain that grows from below $0.05 \%$ to $0.4 \%$ for the final time, this behavior matches what is explained in the previous paragraph.

\subsubsection{Mach 3 tunnel with a step}

REV3: This test case, as described in [43], begins with a Mach 3 flow in a wall-bounded wind tunnel containing a forward step. The tunnel is 3 units long and 1 unit wide, and the step is located at $x=0.2$ with a height of 0.6 units. At the left end there is a inflow boundary condition, and at the right end all gradients are assumed to vanish. Since the flow is supersonic, the exit boundary condition has no effect on the flow. The simulation is carried out until the final time $t=4.0$. This case is used to test the proposed scheme against wall-bounded supersonic flows. Two different grid resolutions are used, namely: 300x100 and 600x200, with a constant $\Delta t$ of $1 E-3$ and 5E-4 respectively. In order to minimize entropy errors, we employ the treatment for the expansion corner described in [44] and we set the epsilon value to 1 on the cells around the expansion corner. Please note that anywhere else, the epsilon value is not fixed and can be modified freely.

REV3: In figures 15 and 16 the obtained results for the two different 
meshes are presented. A numerical Schlieren of the density variable is plotted. The Schlieren values are calculated as

$$
\operatorname{Sch}=\exp \left\{-\beta\left(\frac{|\nabla \rho|-\min |\nabla \rho|}{\max |\nabla \rho|-\min |\nabla \rho|}\right)\right\}
$$

REV3: where $\beta=15$ is the exposure factor and the maximum and minimum are taken over the whole calculable domain, that is, excluding ghost points.

REV3: It is seen that the WENO5 ADA scheme triggers the KelvinHelmholtz instability that occurs around $y=0.8$ in both meshes, which is barely captured by the WENO5 scheme. The results of the WENO5 ADA scheme in the finer grid we observe a fully developed instability which would require a very fine grid for the WENO5 scheme to obtain similar results.
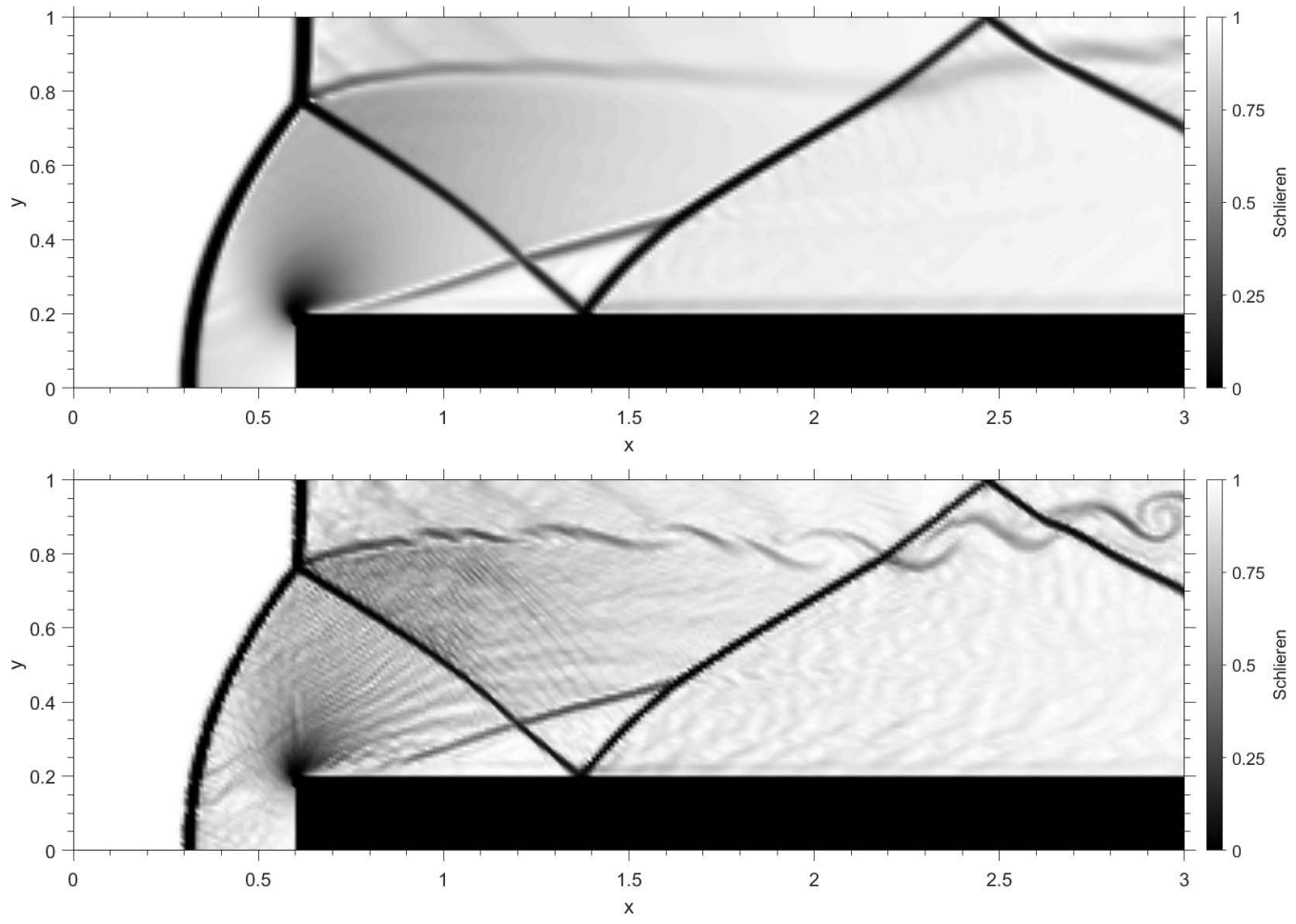

Figure 15: Mach 3 tunnel with a step. WENO5 (top) vs. WENO5 ADA (bottom) density schlieren distribution at $t=4.300 \times 100$ mesh. 

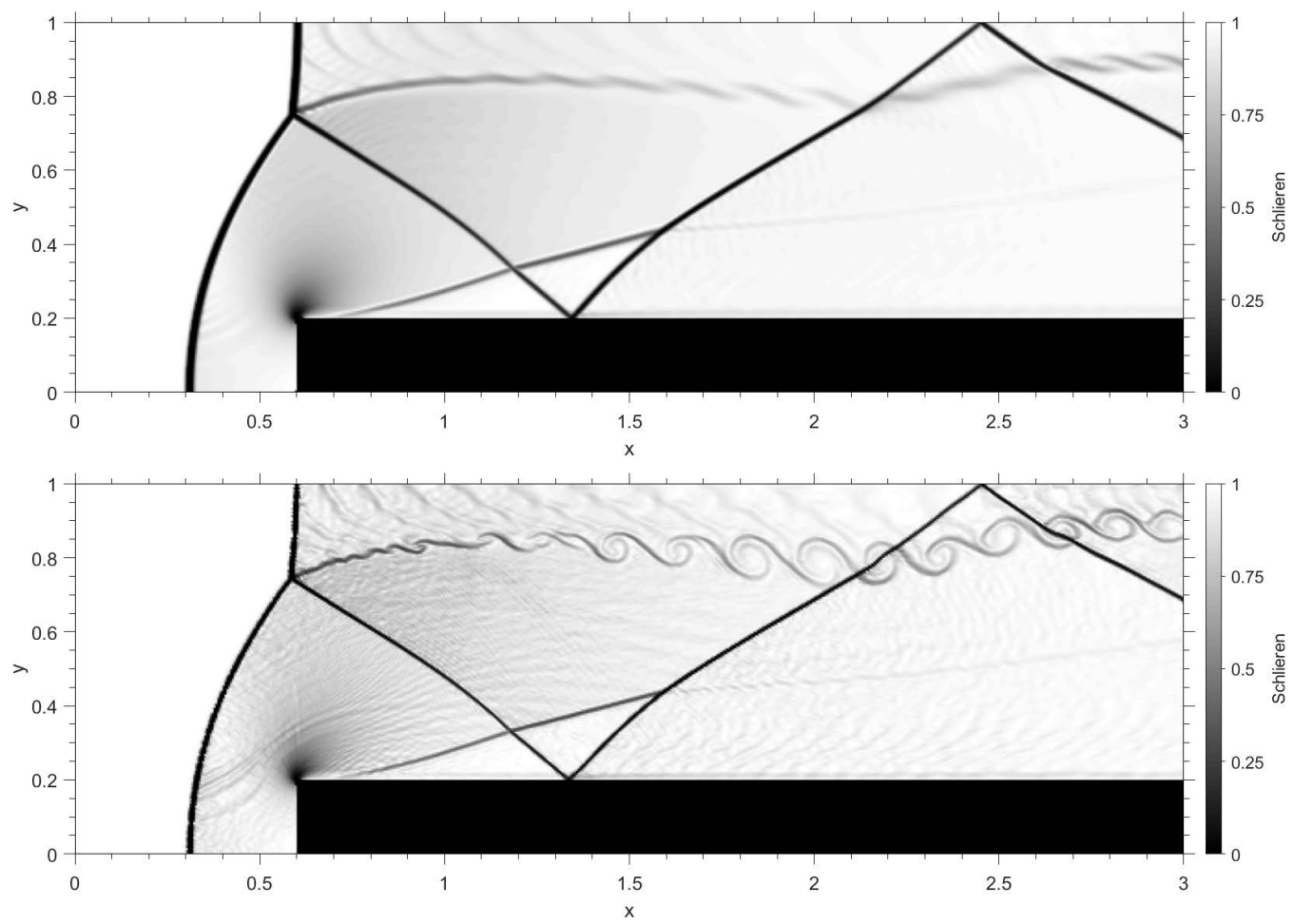

Figure 16: Mach 3 tunnel with a step. WENO5 (top) vs. WENO5 ADA (bottom) density schlieren distribution at $t=4.600 \times 200$ mesh.

REV3: In figure 17, the epsilon value for the final time is plotted for both test meshes. It can be seen that the highest values of epsilon are gathered around shock zones, as expected. 

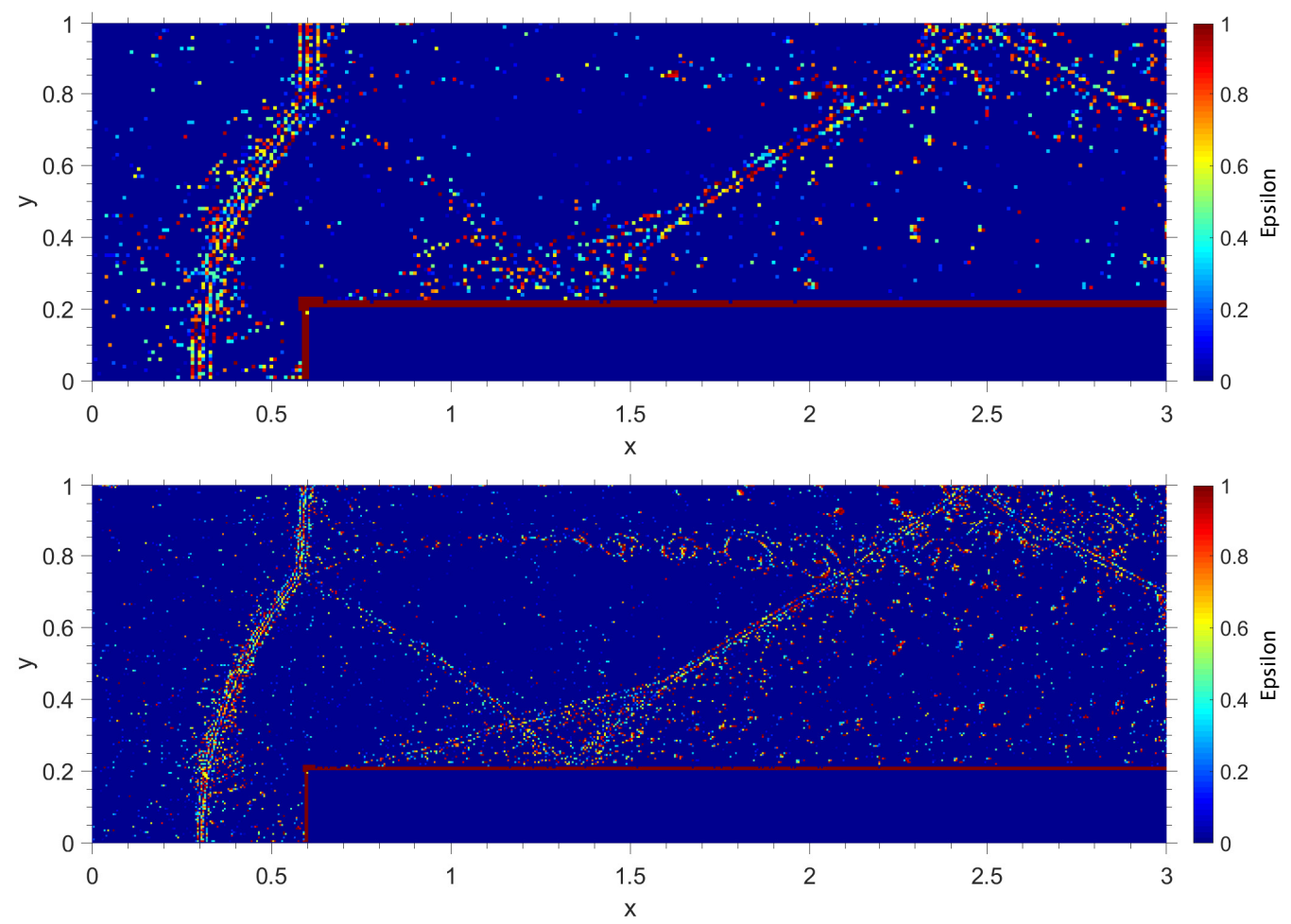

Figure 17: Mach 3 tunnel with a step WENO5 ADA epsilon value distribution at $t=4$. 300x100 mesh (top) vs. 600x200 mesh (bottom). 


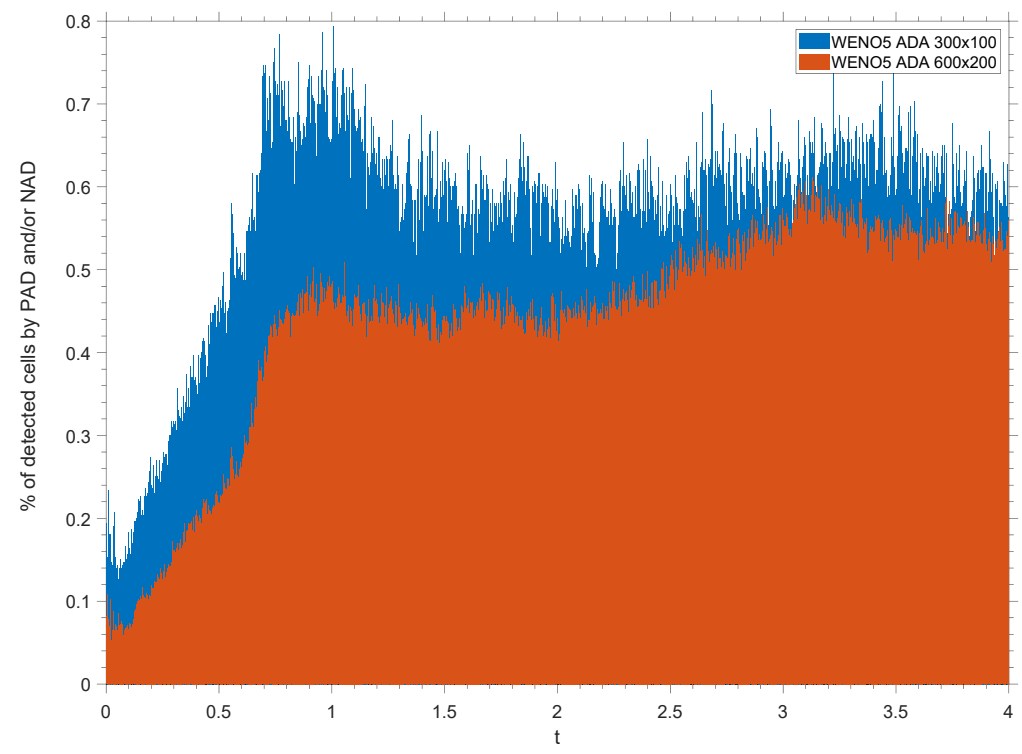

Figure 18: Mach 3 tunnel with a step. WENO5 ADA \% of detected cells during the simulation. 300x100 and 600x200 meshes.

REV3: The evolution of the detected cells along the simulation is shown in figure 10. For this test case, the troubled cells constitute a fraction of the total domain that is under $0.8 \%$.

REV3: In this section we have validated the proposed methodology using two-dimensional benchmarks. Next section will be devoted to study threedimensional test cases.

\subsection{Three-dimensional test cases}

\subsubsection{Incompressible isotropic Taylor-Green vortex}

Incompressible isotropic Taylor-Green vortex The Taylor-Green vortex is the simplest model for the analysis of the nonlinear transfer of kinetic energy among the different scales of the flow. Even if it is simple to construct, it contains several physical processes that are key to understand turbulence. The inviscid version (infinite Reynolds number) of this test case is solved in order to assess the behavior of the proposed method in under-resolved simulations, which are commonly found in industrial computations of turbulent flows at high Reynolds numbers, and to examine the capability of the proposed ILES scheme to reproduce transition to turbulence. For very large Reynolds numbers $(R e \rightarrow \infty)$, it is known that statistically isotropic turbulence of 
incompressible flows develops following the $(-5 / 3)$ decay Kolmogorov's law of the kinetic-energy spectra within the inertial subrange around $t \approx 9$ [45]. A physically consistent numerical method developed for ILES should recover this behavior. In this test case, $\gamma=5 / 3$ is used. The initial conditions of the Taylor-Green vortex are:

$$
\begin{aligned}
\rho(x, y, z, 0) & =1 \\
u(x, y, z, 0) & =\sin (x) \cos (y) \cos (z) \\
v(x, y, z, 0) & =-\cos (x) \sin (y) \cos (z) \\
w(x, y, z, 0) & =0 \\
p(x, y, z, 0) & =100+\frac{1}{16}[(\cos (2 x)+\cos (2 y))(2+\cos (2 z))-2]
\end{aligned}
$$

We solve these equations in a periodic $[0,2 \pi]^{3}$ domain, using a $64^{3}$ grid, until a final time of $t=10$. This grid is used in order to check the behavior of the method for an under-resolved turbulent simulation. Since the density must remain constant in this test case, the kinetic energy is used as the variable to detect oscillations with the a posteriori method.

The evolution of the normalized total kinetic energy and enstrophy using the proposed scheme is shown in figure 19, where the present approach is compared with the results using the unmodified WENO5 scheme.

The non-linear interactions generate successively smaller scales, but the kinetic energy remains constant until $t \approx 4$. The results obtained by the proposed approach, are in excellent agreement with the reference solution, both in terms of the kinetic energy and enstrophy. The use of the ADA method greatly improves the results obtained with the original WENO5 scheme, and it allows to obtain comparable results other schemes presented in the literature [46]. REV1: Even for the coarser grid, the method reproduces the semi-analytical results presented in [47], and gets closer to the $8192^{3}$ mesh results of $[48]$. 

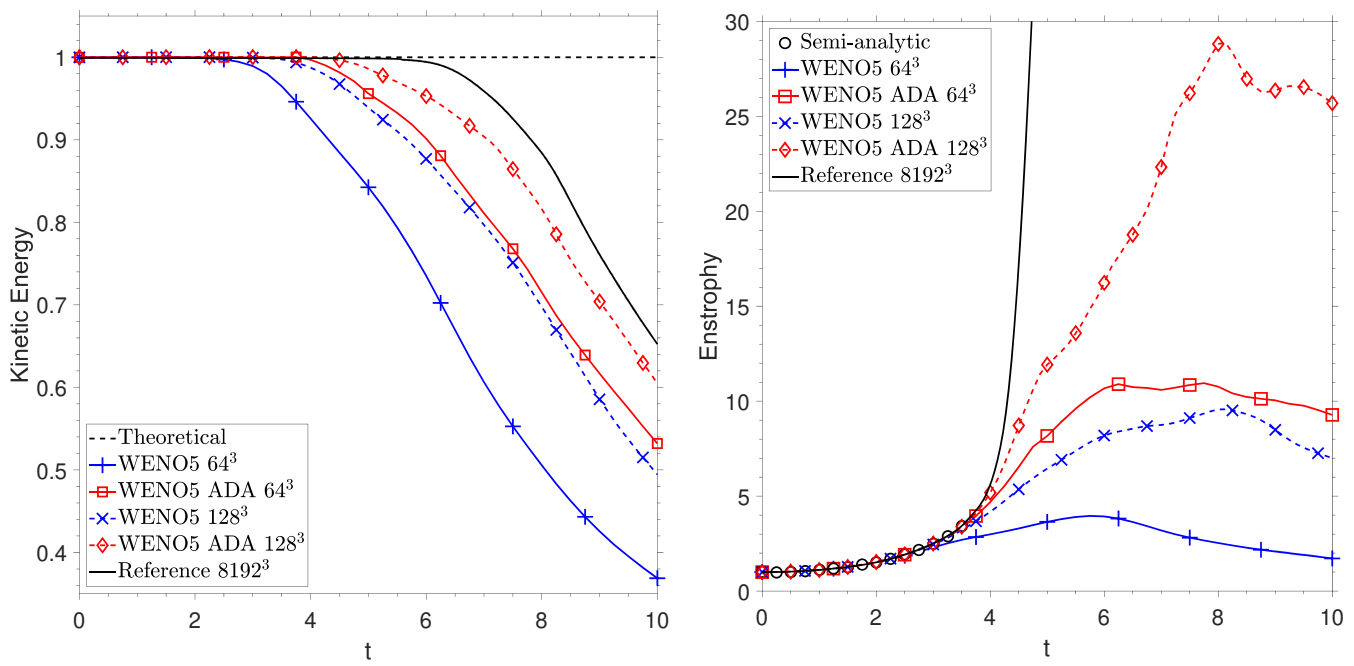

Figure 19: Incompressible isotropic Taylor-Green vortex. Evolution of the normalized kinetic energy (left), evolution of the normalized enstrophy compared with the semi-analytic results of [47] (right). We also present a solution on a very fine grid of $8192^{3}$ digitized from [48] which was computed with a Discontinuous Galerking method. Obtained results for $64^{3}$ - and $128^{3}$-point grids.

In figure 20 the velocity spectrum is shown for the WENO5 and WENO5ADA schemes. The proposed approach, contrary to the unmodified WENO5, approximates really accurately the theoretical slope of $(-5 / 3)$. This can be explained because the WENO5 adds too much dissipation, making the simulation stable but not physical. Indeed, in figure 21 bottom the really different behavior of WENO5 and WENO5 ADA can be seen at an earlier time of $t=5$. The WENO5 ADA approaches the DNS and yields better results than the WENO5. 

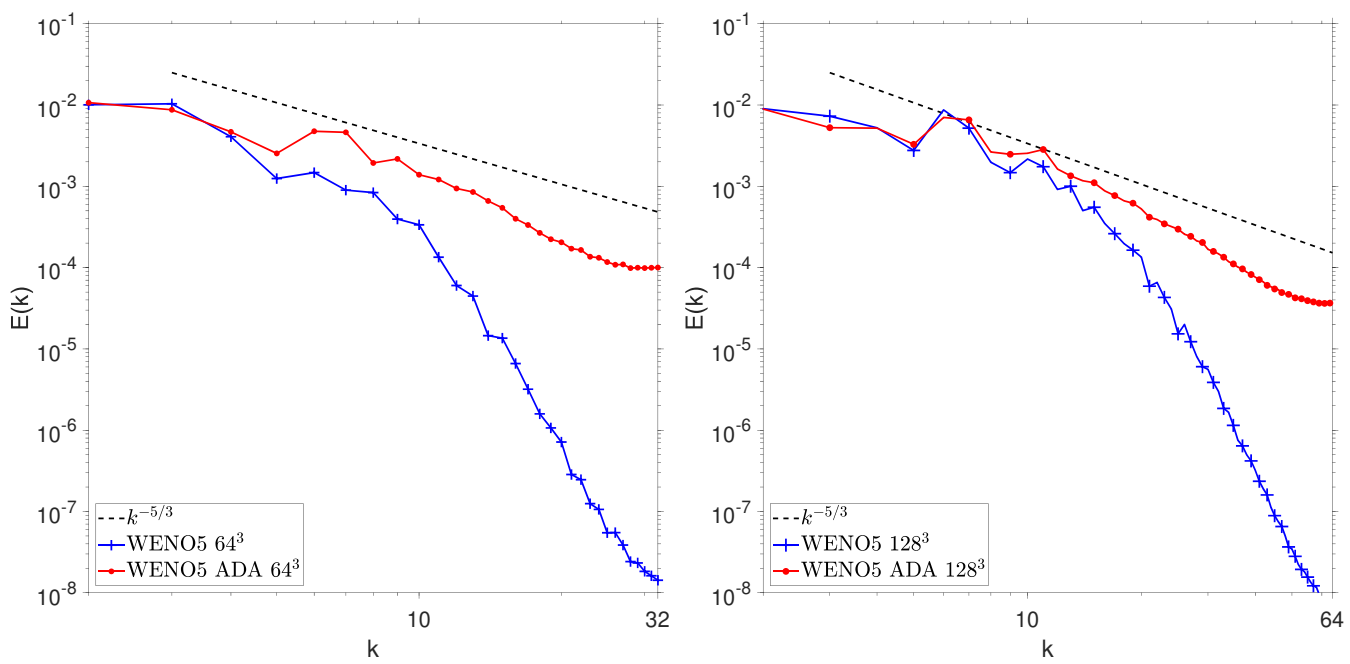

Figure 20: Incompressible isotropic Taylor-Green vortex. Comparative velocity spectrum at $t=10$ for $64^{3}$ and $128^{3}$ point grids.

In figure 21 the vorticity of WENO5 and WENO5 ADA is plotted for two different time instants of $t=3$ and $t=5$, before and after the aforementioned critical time of $t=4$. The results show that for $t=3$ we obtain similar vorticity results, that match the little observable difference on the enstrophy plot of figure 20 , but on $t=5$ the difference is really noticeable. Due to the reduction of viscosity, the vorticity increases. In figure 22 the kinetic energy of WENO5 and WENO5 ADA is plotted for the same times as before. This figure shows that the kinetic energy decay starts later using the WENO5 ADA approach. REV1: This implies that the WENO5 ADA approach starts to introduce additional viscosity when the first subgrid scales appear in the flow at $t \approx 4.1$.

In figures 21 and 22 , a visualization of the vorticity and kinetic energy iso-contours for $t=3$ and $t=5$ is presented for a $64^{3}$-point grid. Once the invisicid part is validated, the next test case will involve the full 3D Navier Stokes equations. 

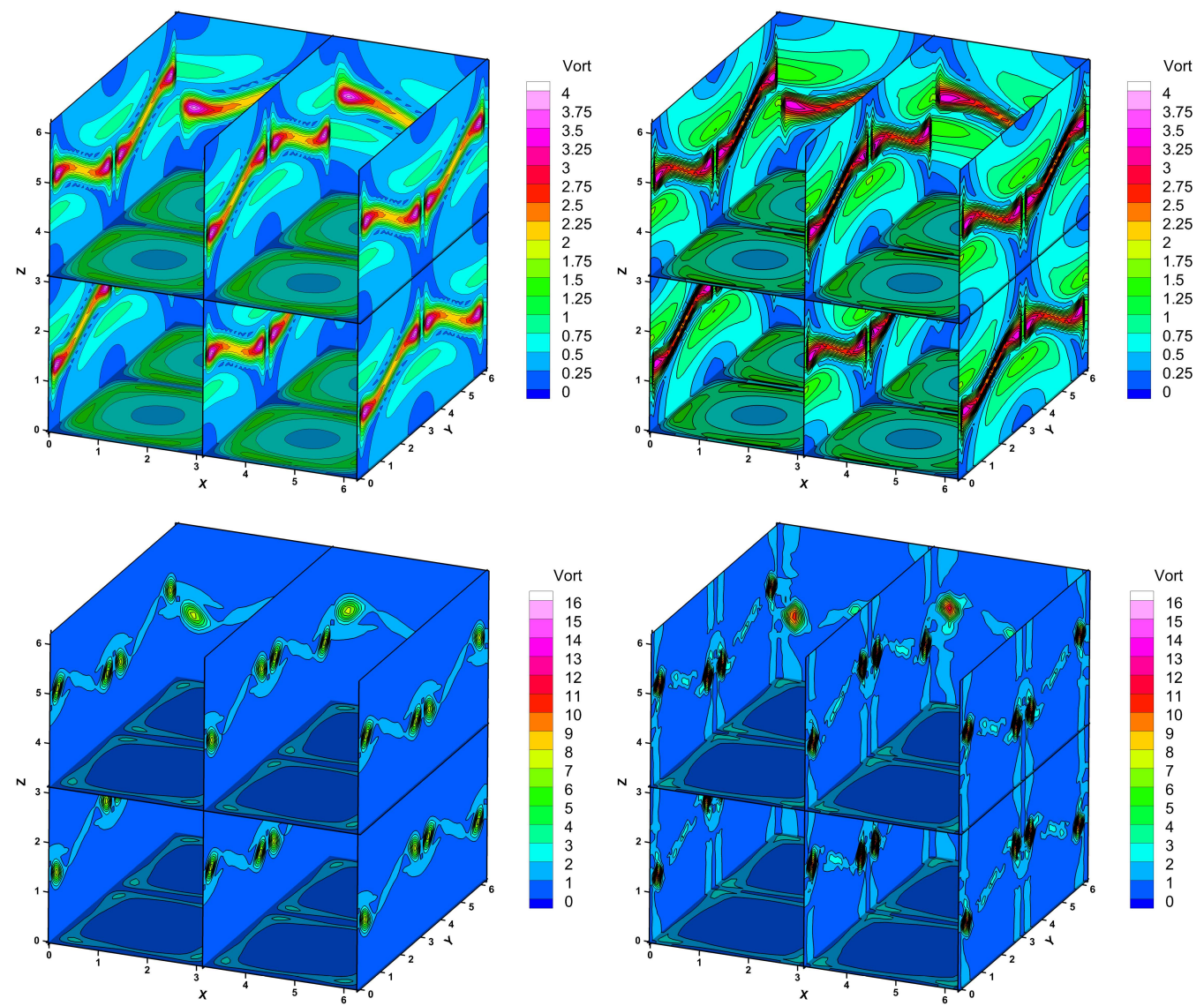

Figure 21: Incompressible isotropic Taylor-Green vortex. Visualization of the vortex stretching through iso-contours of vorticity at $t=3$ (top) and $t=5$ (bottom) for the WENO5 (left) and the WENO5 ADA (right). Obtained results for a $64^{3}$-point grid. 

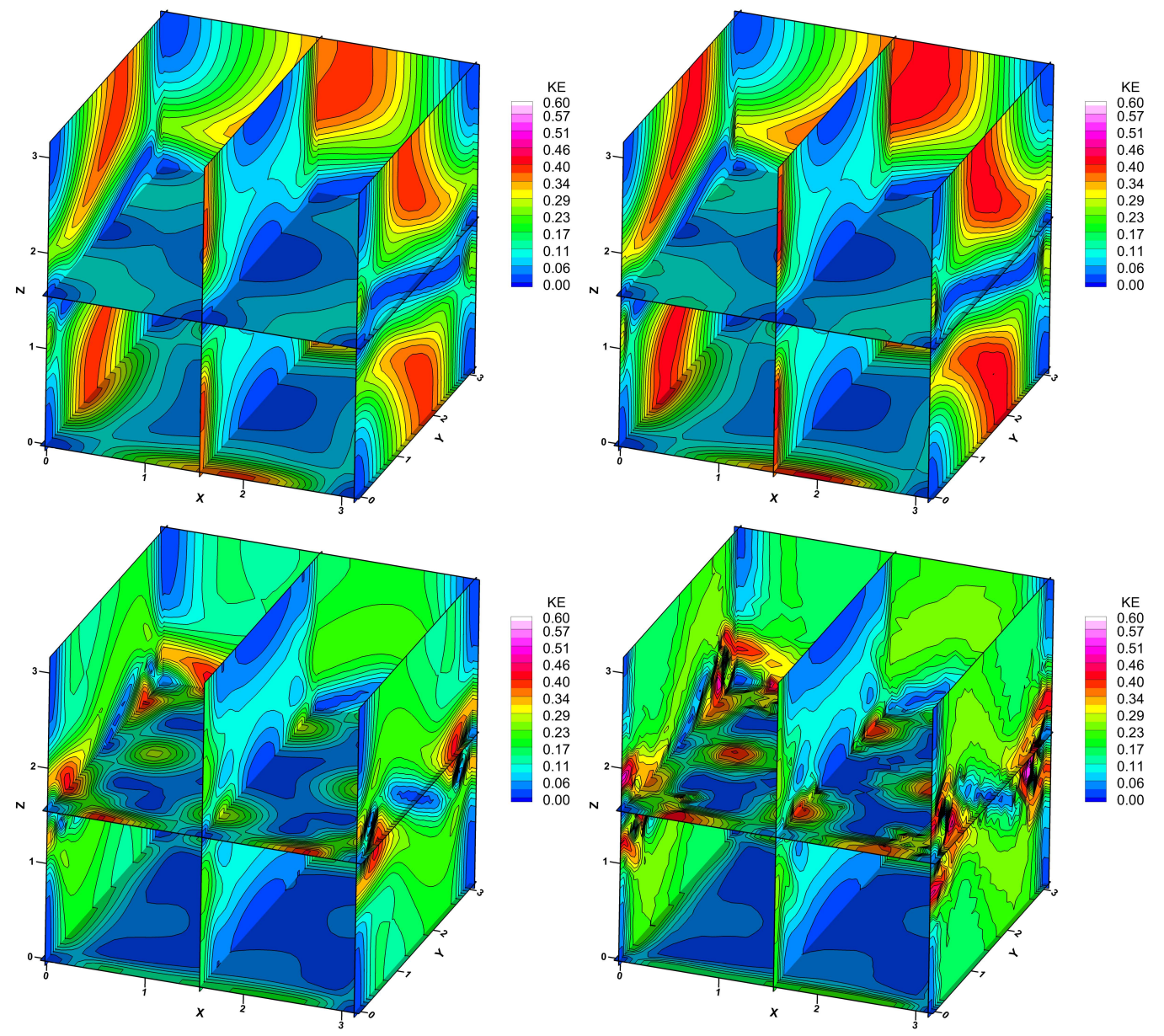

Figure 22: Incompressible isotropic Taylor-Green vortex. Plane cut-away views of the kinetic energy field at $t=3$ (top) and $t=5$ (bottom) for the WENO5 (left) and the WENO5 ADA (right). Obtained results for a $64^{3}$-point grid.

\subsubsection{Incompressible isotropic Taylor-Green vortex with $\mathrm{Re}=1600$}

REV3: In order to analyze the effect of the ADA mechanism on the viscous terms, a finite Reynolds Taylor Green Vortex is also presented. The same domain as in the inviscid version is used, but the initial condition is slightly varied to match the case description provided in [49]. The initial 
condition is set up as

$$
\begin{aligned}
u(x, y, z, 0) & =\sin (x) \cos (y) \cos (z) \\
v(x, y, z, 0) & =-\cos (x) \sin (y) \cos (z) \\
w(x, y, z, 0) & =0 \\
p(x, y, z, 0) & =\frac{1}{\gamma M_{0}^{2}}+\frac{1}{16}[\cos (2 x)+\cos (2 y)][\cos (2 z)+2] \\
\rho(x, y, z, 0) & =\gamma M_{0}^{2} p(x, y, z, 0)
\end{aligned}
$$

REV3: The reference Mach number is chosen as $M_{0}=0.1$ to minimize compressibility effects. A constant Prandtl number of $\operatorname{Pr}=0.71$ and a Reynolds number of $\mathrm{Re}=1600$ are used. The simulation is carried out until $t=10$.
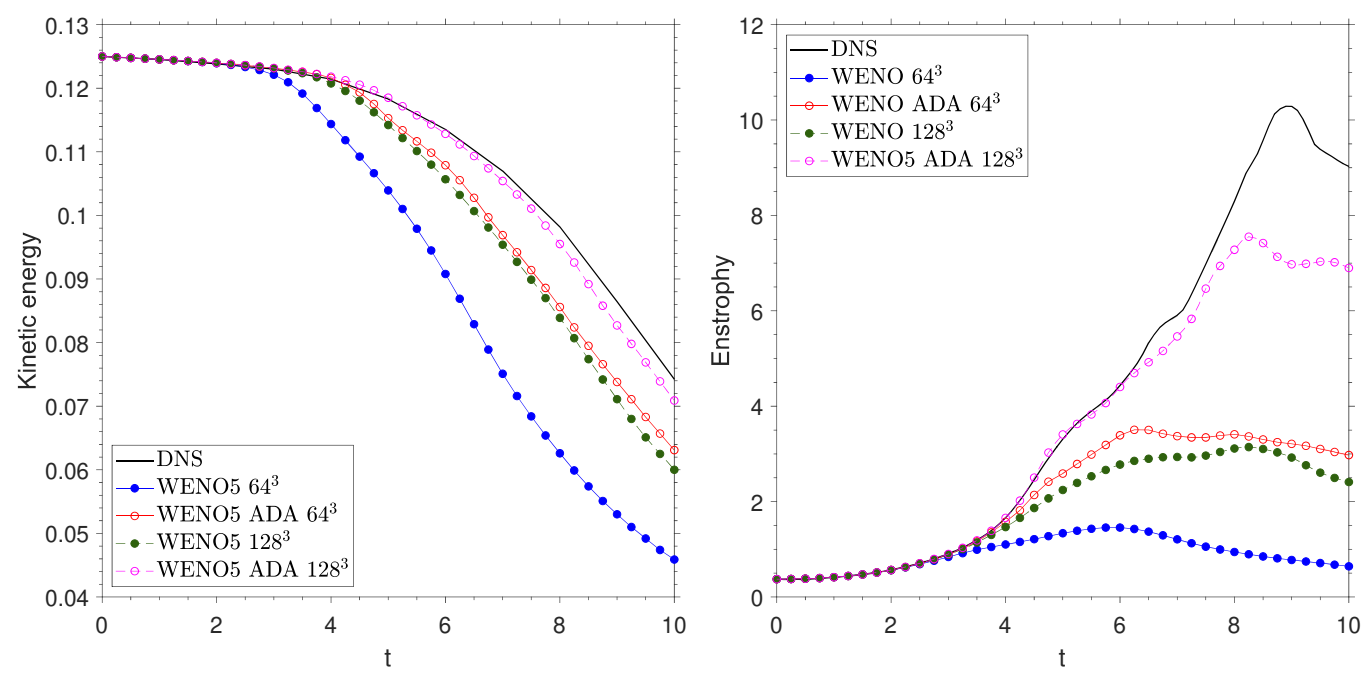

Figure 23: Incompressible isotropic Taylor-Green vortex with $\mathrm{Re}=1600$. Evolution of the kinetic energy (left) and evolution of the enstrophy (right). In both cases the reference DNS solution is digitized from [49].

REV3: In figure 23 the evolution in time of kinetic energy and enstrophy is shown for two meshes, $64^{3}$ and $128^{3}$. It can be seen that the ADA procedure on the coarser mesh outperforms the original WENO in the finer mesh, while being in fairly good agreement with the reference solution for the finer mesh. In both cases, we obtain remarkable improvements with respect to the original approach. 


\subsubsection{Decay of compressible homogeneous isotropic turbulence}

This test is a widely used benchmark $[50,51,52,53]$ that models a simple viscous turbulent flow, but it allows to assess the ability of the numerical scheme for Large Eddy Simulations (LES). The problem takes place in a periodic $[0,2 \pi]^{3}$ domain. The initial three-dimensional kinetic energy spectrum is defined as

$$
E(k) \sim k^{4} \exp \left(-2\left(\frac{k}{k_{0}}\right)^{2}\right)
$$

where $k$ is the magnitude of the wave number vector, and $k_{0}=4$ is the wavenumber at the peak of the spectrum. Using this initial energy spectrum, $\lambda_{0}=0.5 k_{0}$. Two different configurations will be tested in order to assess the performance of the method.

\section{HIT1 test case}

The first test case, referred to as HIT1, and is taken from $[51,53]$. Initial velocity fluctuations are parametrized by the turbulent Mach number, and also by the fraction of energy in the dilatational part of the velocity, $\chi=0.2$ [54]. The initial turbulent Mach number is taken as $M_{t_{0}}=0.4$ and the initial Taylor microscale Reynolds number $\operatorname{Re}_{\lambda_{0}}=2157$, which corresponds to a Reynolds number $\mathrm{Re}=536.9$ [55]. The initial density and temperature fields are given by:

$$
\begin{aligned}
\left(\rho_{\text {rms }}^{\prime}\right)^{2} /\langle\rho\rangle^{2} & =0.032 \\
\left(T_{\text {rms }}^{\prime}\right)^{2} /\langle T\rangle^{2} & =0.005
\end{aligned}
$$

The symbol \langle\rangle refers to mean value and primes denote fluctuating variables. Subscript rms stands for root mean square. In the case of the velocity, we define $v_{\text {rms }}$ as

$$
v_{\mathrm{rms}}=\sqrt{\frac{\left\langle v_{i}^{\prime} v_{i}^{\prime}\right\rangle}{3}}
$$

Two different grids of $32^{3}$ and $64^{3}$ points are used. The time stepping for the coarser grid is $\Delta t=0.05$ which corresponds to 300 iterations until the final eddy turnover time $t / \tau=1.2$. REV2: For the finer grid $\Delta t=0.025$ is used, which corresponds to 600 iterations.. 
These grids correspond to under-resolved simulations, since we are interested in the behavior of the scheme in LES simulations. The results are compared with a reference solution computed with a sixth-order compact Finite Difference scheme, with explicit filtering using a tenth-order Padé filter [56]. This solution closely follows the DNS results presented in [29].
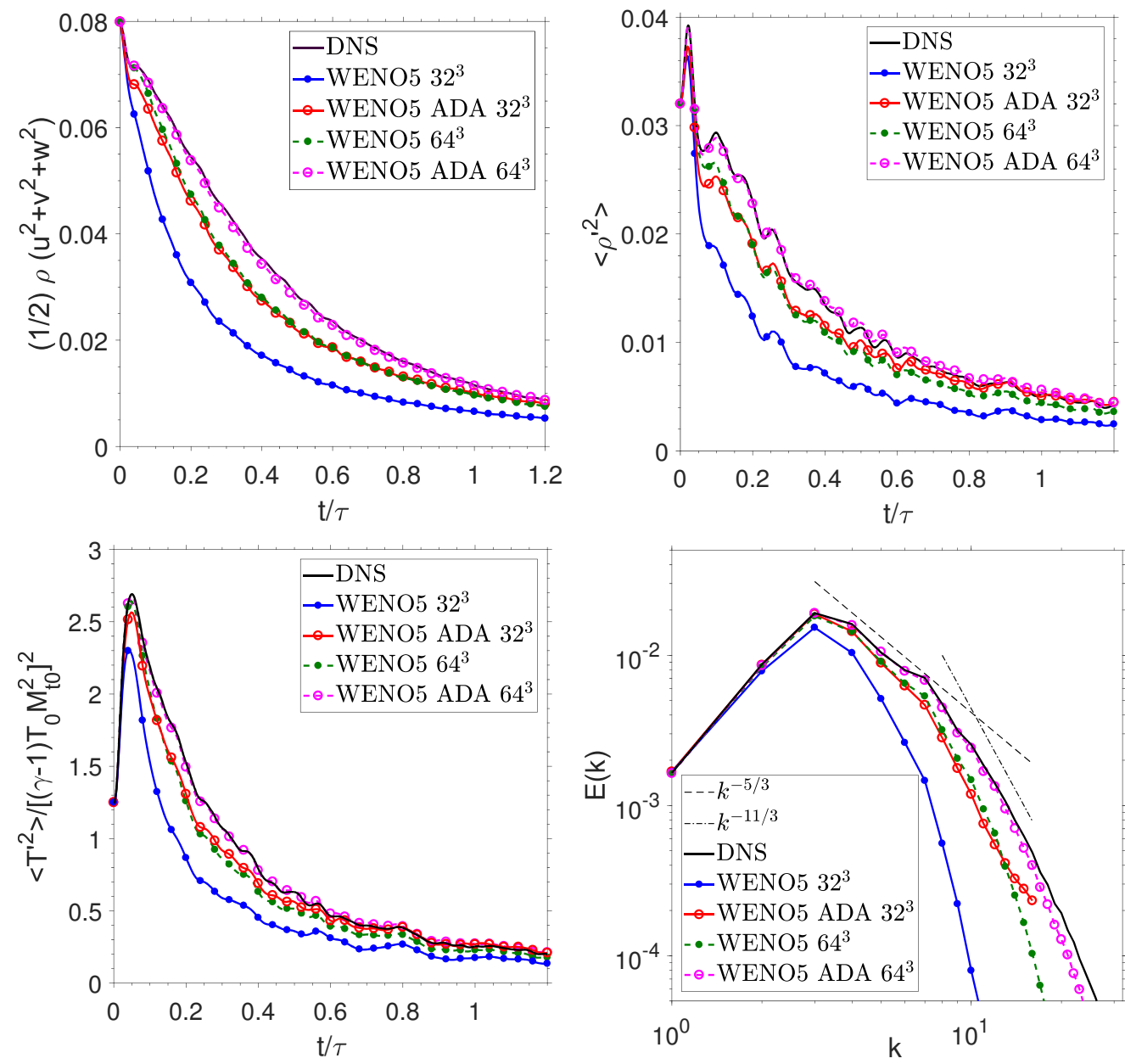

Figure 24: Decay of compressible homogeneous isotropic turbulence. HIT1 case. Evolution of kinetic energy (top left), mean-square density fluctuations (top right), normalized meansquare temperature fluctuations (bottom left) and energy spectrum (bottom right) for a $32^{3}$ - and $64^{3}$-point grids. DNS results obtained by using a $128^{3}$-point grid with a sixthorder compact differences plus a tenth-order filter as in [29].

In figure 24 the results for this test case are plotted. A real improvement 
of the WENO5 ADA scheme is observed, achieving even better results than those of the WENO5 on a finer grid. For the finest $64^{3}$-point grid, the results follow the DNS really closely over time for all plotted variables. Concerning the energy spectrum, the two different slopes that were described earlier are captured by the numerical simulation. Thus, the slope $k^{-5 / 3}$ holds on the inertial range and $k^{-11 / 3}$ holds on the smallest scales. It is seen that the results obtained by both numerical schemes follow these slopes, but the WENO5 ADA achieves closer results to the DNS on the finer grid, and on the coarser grid performs slightly better than the WENO5 on the finer grid.

\section{HIT2 test case}

The second configuration is taken from $[46,57]$. The initial flow parameters are $\operatorname{Re}_{\lambda_{0}}=100$ and $M_{t_{0}}=0.6$. In this case the density and pressure fields are initially constant. We take $\rho=1$ and the pressure is computed accordingly.

This setup corresponds to the nonlinear subsonic regime and weak shocklets develop spontaneously from the turbulent motion. This fact poses a challenge to the accuracy of any numerical scheme, as explained in $[46,57]$.

In figure 25 the results of the WENO5 and WENO5 ADA are plotted along with the results for the DNS from [46]. For this test case, the WENO5 ADA scheme is in really good agreement with the DNS, and greatly outperforms the results obtained by the original scheme. It is remarkable the excellent agreement between the results of the proposed WENO ADA scheme and the DNS solutions for the mean-square velocity, enstrophy, dilatation and temperature fluctuations. We note the excellent behavior of the proposed scheme to reproduce the thermodynamic variables of the flow.

The energy spectra for the WENO ADA and the original WENO5 schemes are plotted in figure 26. Using the proposed scheme, the obtained spectrum closely follows that of the DNS in the whole range of scales under the Nyquist frequency. 

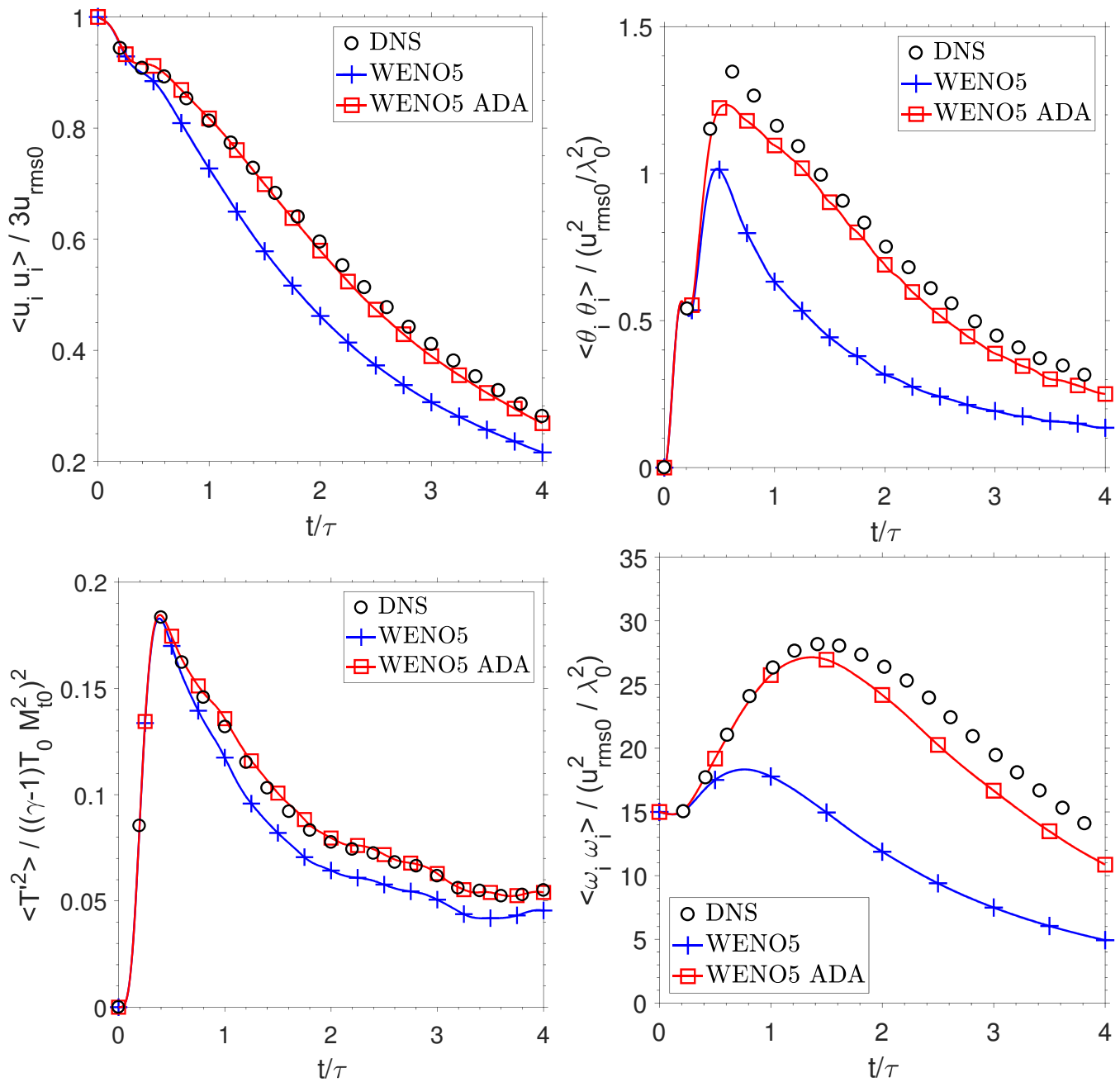

Figure 25: Decay of compressible homogeneous isotropic turbulence. HIT2 case. Evolution of mean-square velocity (top left), dilatation (top right), normalized mean-square temperature fluctuations (bottom left) and enstrophy (bottom right) for a $64^{3}$-point grid. DNS results digitized from [46]. 


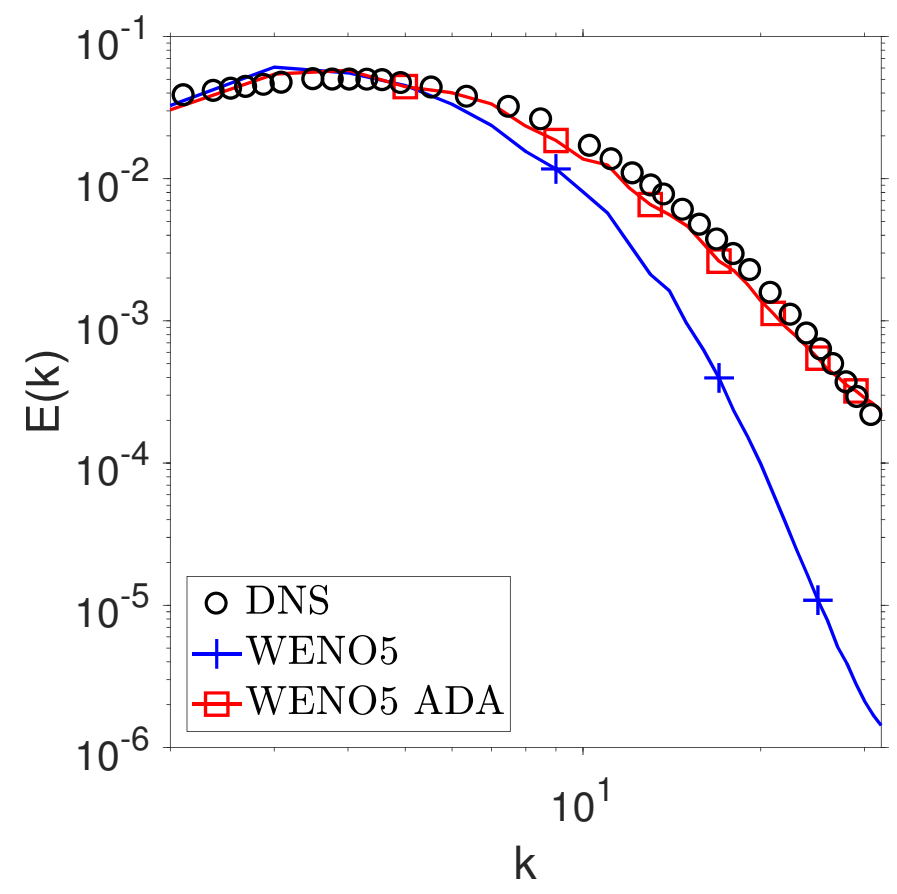

Figure 26: Decay of compressible homogeneous isotropic turbulence. HIT2 case. Instantaneous three-dimensional velocity spectrum at $t / \tau=4$ for a $64^{3}$-point grid. DNS results digitized from [46].

\section{Conclusions}

In this work a coupling of the Adaptive Dissipation Adjustment (ADA) with the WENO5 numerical scheme is proposed in conjunction with the a posteriori paradigm. This is achieved by separating the WENO reconstruction into a central and a dissipative part and inserting a multiplicative coefficient $\varepsilon \in[0,1]$ on the latter. This coefficient regulates the amount of dissipation introduced in the inviscid flux reconstruction, adjusting its value by evaluating the content of high frequencies of the solution with the aid of the energy ratio. It achieves similar results to the original scheme in the onedimensional test cases that have been presented, and greatly improves the results for the turbulent three-dimensional ones. By looking at the WENO5 results, it is clearly seen that the stability of the scheme does not necessarily imply any physical correctness at all, since the WENO5 results, although numerically stable, does not exhibit the physical features of the DNS results. On the contrary, the obtained results of the WENO5 ADA method are in 
really good agreement with the DNS, outperforming other reference schemes for some of the test cases.

The present formulation, although it is explained for the WENO5 scheme, it can be readily extended to other members of the WENO family, the only changes being the function $\varphi_{N}$ of equation (33) and the central scheme that will be applied.

\section{Acknowledgements}

This work has been partially supported by the Ministerio de Ciencia, Innovación y Universidades (grant \#RTI2018-093366-B-I00) of the Spanish Government and by the Consellería de Educación e Ordenación Universitaria of the Xunta de Galicia (grant \#ED431C 2018/41), cofinanced with FEDER funds and the Universidade da Coruña. Xesús Nogueira also acknowledges the funding provided by the Xunta de Galicia through the program Axudas para a mellora, creación, recoñecemento e estruturación de agrupacións estratéxicas do Sistema universitario de Galicia (reference \# ED431E 2018/11). 


\section{References}

[1] Harten A, Engquist B, Osher S, Chakravarthy SR. Uniformly high order accurate essentially non-oscillatory schemes, III. Journal of Computational Physics. 1987 Aug;71(2):231-303.

[2] N EH. The Calculus of Finite Differences. Nature. 1934 Aug;134(3381):231-233.

[3] Jiang GS, Shu CW. Efficient Implementation of Weighted ENO Schemes. Journal of Computational Physics. 1996 Jun;126(1):202-228.

[4] Dumbser M. Arbitrary-Lagrangian-Eulerian ADER-WENO finite volume schemes with time-accurate local time stepping for hyperbolic conservation laws. Computer Methods in Applied Mechanics and Engineering. 2014 oct;280:57-83.

[5] Dumbser M, Hidalgo A, Zanotti O. High order space-time adaptive ADER-WENO finite volume schemes for non-conservative hyperbolic systems. Computer Methods in Applied Mechanics and Engineering. 2014 jan;268:359-387.

[6] Balsara DS, Shu CW. Monotonicity Preserving Weighted Essentially Non-oscillatory Schemes with Increasingly High Order of Accuracy. Journal of Computational Physics. 2000 May;160(2):405-452.

[7] Henrick AK, Aslam TD, Powers JM. Mapped weighted essentially non-oscillatory schemes: Achieving optimal order near critical points. Journal of Computational Physics. 2005 Aug;207(2):542-567.

[8] Borges R, Carmona M, Costa B, Don WS. An improved weighted essentially non-oscillatory scheme for hyperbolic conservation laws. Journal of Computational Physics. 2008 Mar;227(6):3191-3211.

[9] Castro M, Costa B, Don WS. High order weighted essentially non-oscillatory WENO$\mathrm{Z}$ schemes for hyperbolic conservation laws. Journal of Computational Physics. 2011 Mar;230(5):1766-1792.

[10] Acker F, de R Borges RB, Costa B. An improved WENO-Z scheme. Journal of Computational Physics. 2016 May;313:726-753.

[11] Hill DJ, Pullin DI. Hybrid tuned center-difference-WENO method for large eddy simulations in the presence of strong shocks. Journal of Computational Physics. 2004 Mar;194(2):435-450.

[12] Weirs V, Candler G, Weirs V, Candler G. Optimization of weighted ENO schemes for DNS of compressible turbulence. In: 13th Computational Fluid Dynamics Conference. American Institute of Aeronautics and Astronautics; 1997. p. 528-538.

[13] Arshed GM, Hoffmann KA. Minimizing errors from linear and nonlinear weights of WENO scheme for broadband applications with shock waves. Journal of Computational Physics. 2013 Aug;246:58-77. 
[14] Hu XY, Tritschler VK, Pirozzoli S, Adams NA. Dispersion-dissipation condition for finite difference schemes; 2012. arXiv:1204.5088 [physics.flu-dyn].

[15] Hu XY, Wang Q, Adams NA. An adaptive central-upwind weighted essentially nonoscillatory scheme. Journal of Computational Physics. 2010 Nov;229(23):8952-8965.

[16] $\mathrm{Hu} \mathrm{X}$, Adams N. Scale separation for implicit large eddy simulation. Journal of Computational Physics. 2011 Aug;230(19):7240-7249.

[17] Fu L, Hu XY, Adams NA. A family of high-order targeted ENO schemes for compressible-fluid simulations. Journal of Computational Physics. 2016 Jan;305:333359 .

[18] Fu L, Hu XY, Adams NA. Targeted ENO schemes with tailored resolution property for hyperbolic conservation laws. Journal of Computational Physics. 2017 Nov;349:97121.

[19] Fu L. A Hybrid Method with TENO Based Discontinuity Indicator for Hyperbolic Conservation Laws. Communications in Computational Physics. 2019 jun;26(4):9731007.

[20] Pirozzoli S. Conservative Hybrid Compact-WENO Schemes for Shock-Turbulence Interaction. Journal of Computational Physics. 2002 may;178(1):81-117.

[21] Costa B, Don WS. High order Hybrid central-WENO finite difference scheme for conservation laws. Journal of Computational and Applied Mathematics. 2007 jul;204(2):209-218.

[22] Fernández-Fidalgo J, Nogueira X, Ramírez L, Colominas I. An a posteriori, efficient, high-spectral resolution hybrid finite-difference method for compressible flows. Computer Methods in Applied Mechanics and Engineering. 2018 jun;335:91-127.

[23] Balsara DS, Garain S, Shu CW. An efficient class of WENO schemes with adaptive order. Journal of Computational Physics. 2016 dec;326:780-804.

[24] Ghosh D, Baeder JD. Compact Reconstruction Schemes with Weighted ENO Limiting for Hyperbolic Conservation Laws. SIAM Journal on Scientific Computing. 2012 jan;34(3):A1678-A1706.

[25] Domaradzki JA, Loh KC, Yee PP. Large Eddy Simulations Using the Subgrid-Scale Estimation Model and Truncated Navier-Stokes Dynamics. Theoretical and Computational Fluid Dynamics. 2002 Jul;15(6):421-450.

[26] Tantikul T, Domaradzki JA. Large eddy simulations using truncated Navier-Stokes equations with the automatic filtering criterion. Journal of Turbulence. 2010 Jan;11:N21. 
[27] Clain S, Diot S, Loubère R. A high-order finite volume method for systems of conservation laws-Multi-dimensional Optimal Order Detection (MOOD). Journal of Computational Physics. 2011 May;230(10):4028-4050.

[28] Diot S, Clain S, Loubère R. Improved detection criteria for the Multi-dimensional Optimal Order Detection (MOOD) on unstructured meshes with very high-order polynomials. Computers \& Fluids. 2012 Jul;64:43-63.

[29] Visbal MR, Rizzetta DP. Large-Eddy Simulation on Curvilinear Grids Using Compact Differencing and Filtering Schemes. Journal of Fluids Engineering. 2002;124(4):836.

[30] Shu CW, Osher S. Efficient implementation of essentially non-oscillatory shockcapturing schemes, II. Journal of Computational Physics. 1989 Jul;83(1):32-78.

[31] Shu CW. Essentially non-oscillatory and weighted essentially non-oscillatory schemes for hyperbolic conservation laws. In: Lecture Notes in Mathematics. Springer Berlin Heidelberg; 1998. p. 325-432.

[32] Jiang GS, Wu C. A High-Order WENO Finite Difference Scheme for the Equations of Ideal Magnetohydrodynamics. Journal of Computational Physics. 1999 Apr;150(2):561-594.

[33] Nogueira X, Ramírez L, Fernández-Fidalgo J, Deligant M, Khelladi S, Chassaing JC, et al. An a posteriori-implicit turbulent model with automatic dissipation adjustment for Large Eddy Simulation of compressible flows. Computers \& Fluids. 2020 Jan;197:104371.

[34] Lancaster P, Salkauskas K. Surfaces generated by moving least squares methods. Mathematics of Computation. 1981 Sep;37(155):141-141.

[35] Clain S, Figueiredo J, Loubère R. An Overview on the Multidimensional Optimal Order Detection Method. SYMCOMP 2015. 2015.

[36] Diot S, Loubère R, Clain S. The Multidimensional Optimal Order Detection method in the three-dimensional case: very high-order finite volume method for hyperbolic systems. International Journal for Numerical Methods in Fluids. 2013 may;73(4):362392.

[37] Figueiredo J, Clain S. Second-order Finite Volume Mood Method for the Shallow Water with Dry/wet Interface. SYMCOMP 2015. 2015.

[38] Dumbser M, Zanotti O, Loubère R, Diot S. A posteriori subcell limiting of the discontinuous Galerkin finite element method for hyperbolic conservation laws. Journal of Computational Physics. 2014 Dec;278:47-75.

[39] Shen Y, Wang B, Zha G. Implicit WENO Scheme and High Order Viscous Formulas for Compressible Flows. In: 25th AIAA Applied Aerodynamics Conference. American Institute of Aeronautics and Astronautics; 2007. . 
[40] Toro EF. Riemann Solvers and Numerical Methods for Fluid Dynamics. Springer Berlin Heidelberg; 1999.

[41] Masatsuka K. I do like CFD, VOL.1, Second Edition. v. 1. K. Masatsuka; 2013.

[42] Lax PD, Liu XD. Solution of Two-Dimensional Riemann Problems of Gas Dynamics by Positive Schemes. SIAM Journal on Scientific Computing. 1998 mar;19(2):319340 .

[43] Woodward P, Colella P. The numerical simulation of two-dimensional fluid flow with strong shocks. Journal of Computational Physics. 1984 Apr;54(1):115-173.

[44] Botta N, Jeltsch R. A numerical method for unsteady flows. Applications of Mathematics. 1995;40(3):175-201.

[45] Fauconnier D, Langhe CD, Dick E. Construction of explicit and implicit dynamic finite difference schemes and application to the large-eddy simulation of the Taylor-Green vortex. Journal of Computational Physics. 2009 Nov;228(21):8053-8084.

[46] Johnsen E, Larsson J, Bhagatwala AV, Cabot WH, Moin P, Olson BJ, et al. Assessment of high-resolution methods for numerical simulations of compressible turbulence with shock waves. Journal of Computational Physics. 2010 Feb;229(4):1213-1237.

[47] Brachet ME, Meiron DI, Orszag SA, Nickel BG, Morf RH, Frisch U. Small-scale structure of the Taylor-Green vortex. Journal of Fluid Mechanics. 1983 May;130(1):411.

[48] Fehn N, Kronbichler M, Munch P, Wall WA. Numerical evidence of anomalous energy dissipation in incompressible Euler flows: Towards grid-converged results for the inviscid Taylor-Green problem. arXiv 200701656. 2020.

[49] Garmann DJ, Visbal MR. AFRL Contributions to the Third International Workshop on High-Order CFD Methods. Third International Workshop on High-Order CFD Methods; 2015. .

[50] Lee S, Lele SK, Moin P. Eddy shocklets in decaying compressible turbulence. Physics of Fluids A: Fluid Dynamics. 1991 Apr;3(4):657-664.

[51] Spyropoulos ET, Blaisdell GA. Evaluation of the dynamic model for simulations of compressible decaying isotropic turbulence. AIAA Journal. 1996 May;34(5):990-998.

[52] Honein AE, Moin P. Higher entropy conservation and numerical stability of compressible turbulence simulations. Journal of Computational Physics. 2004 Dec;201(2):531545 .

[53] Nogueira X, Khelladi S, Colominas I, Cueto-Felgueroso L, París J, Gómez H. HighResolution Finite Volume Methods on Unstructured Grids for Turbulence and Aeroacoustics. Archives of Computational Methods in Engineering. 2011 jul;18(3):315-340. 
[54] Sarkar S, Erlebacher G, Hussaini MY, Kreiss HO. The analysis and modelling of dilatational terms in compressible turbulence. Journal of Fluid Mechanics. 1991 Jun;227(-1):473.

[55] Rizzetta D, Visbal M, Blaisdell G. Application of a high-order compact difference scheme to large-eddy and direct numerical simulation. In: 30th Fluid Dynamics Conference. American Institute of Aeronautics and Astronautics; 1999. .

[56] Visbal MR, Gaitonde DV. High-Order Schemes for Navier-Stokes Equations: Algorithm and Implementation Into FDL3DI. Air Force Research Lab Wright-Patterson, Air Vehicles Directorate; 1998.

[57] Kotov DV, Yee HC, Wray AA, Sjögreen B, Kritsuk AG. Numerical dissipation control in high order shock-capturing schemes for LES of low speed flows. Journal of Computational Physics. 2016 Feb;307:189-202. 
2020-08-04

\section{A reduced-dissipation WENO scheme with automatic dissipation adjustment}

Fernández-Fidalgo, Javier

Elsevier

Fernández-Fidalgo J, Ramírez L, Tsoutsanis $\mathrm{P}$, et al., (2021) A reduced-dissipation WENO

scheme with automatic dissipation adjustment. Journal of Computational Physics, Volume 425, January 2021, Article number 109749

https://doi.org/10.1016/j.jcp.2020.109749

Downloaded from Cranfield Library Services E-Repository 